\title{
Symplectic connections
}

\author{
Pierre Bieliavsky ${ }^{1}$
}

Michel Cahen ${ }^{2}$

Simone Gutt ${ }^{2,3}$

John Rawnsley ${ }^{4}$

Lorenz Schwachhöfer ${ }^{5}$

\begin{abstract}
This article is an overview of the results obtained in recent years on symplectic connections. We present what is known about preferred connections (critical points of a variational principle). The class of Ricci-type connections (for which the curvature is entirely determined by the Ricci tensor) is described in detail, as well as its far reaching generalization to special connections. A twistorial construction shows a relation between Ricci-type connections and complex geometry. We give a construction of Ricci-flat symplectic connections. We end up by presenting, through an explicit example, an approach to noncommutative symplectic symmetric spaces.
\end{abstract}

math.SG/0511194 v2, May 2006. Section 6.8 rewritten.

\footnotetext{
${ }^{1}$ Univ. Cath. Louvain, Dépt de Math, ch. du cyclotron 2, B-1348 Louvain-la-Neuve, Belgium

${ }^{2}$ Université Libre de Bruxelles, Campus Plaine, CP 218, B-1050 Brussels, Belgium

${ }^{3}$ Université de Metz, Dépt. de Math., Ile du Saulcy, F-57045 Metz Cedex 01, France

${ }^{4}$ Mathematics Institute, University of Warwick, Coventry CV4 7AL, United Kingdom

${ }^{5}$ Math. Institut, Universität Dortmund, Vogelpothsweg 87, D-44221 Dortmund, Germany

bieliavsky@math.ucl.ac.be, mcahen@ulb.ac.be, sgutt@ulb.ac.be,

j.rawnsley@warwick.ac.uk, lschwach@math.uni-dortmund.de
} 


\section{Contents}

1 Introduction 1

2 Definitions and basic facts about symplectic connections 1

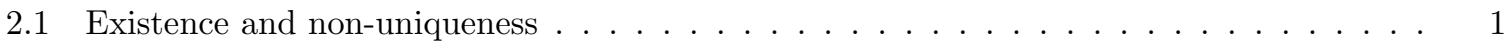

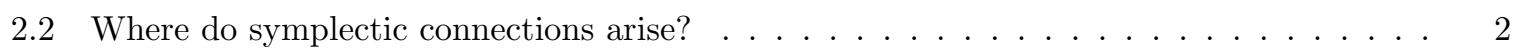

2.3 When is there a "natural" unique symplectic connection? . . . . . . . . . . . 3

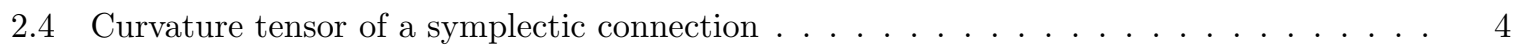

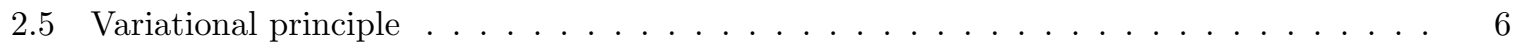

3 Preferred symplectic connections $\quad 6$

3.1 Preferred symplectic connections in dimension $2 \ldots \ldots \ldots \ldots \ldots$

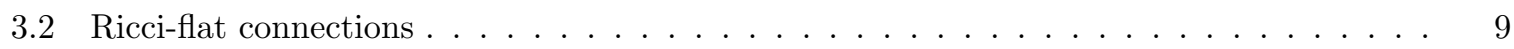

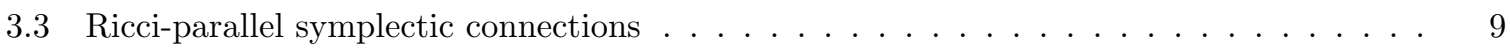

3.4 Homogeneous preferred connections . . . . . . . . . . . . . . . . . . . . . . 10

4 Ricci-type connections $\quad 11$

4.1 Some properties of the curvature of a Ricci-type connection . . . . . . . . . . . 11

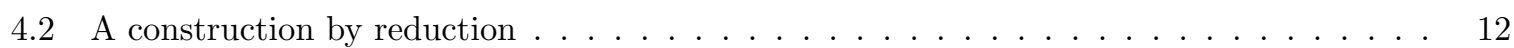

4.3 Local models for Ricci-type connections . . . . . . . . . . . . . . . . . . . . 13

4.4 Global models for Ricci-type connections . . . . . . . . . . . . . . . . . . . 14

5 Special symplectic connections $\quad 14$

6 Symplectic twistor space and Ricci-type connections 19

6.1 Compatible almost complex structures . . . . . . . . . . . . . . . . . . 19

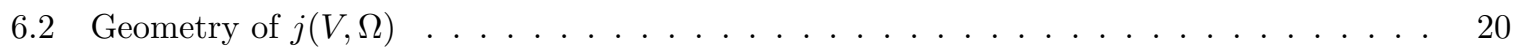

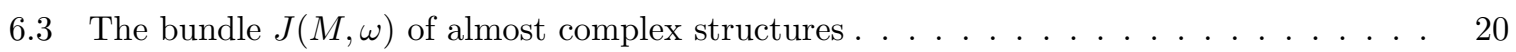

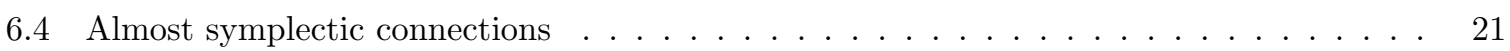

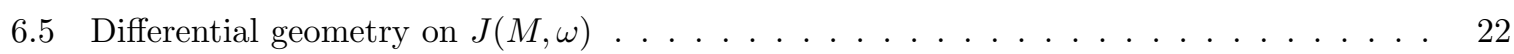

6.6 The almost complex structures on $J(M, \omega) \ldots \ldots \ldots \ldots \ldots \ldots \ldots$

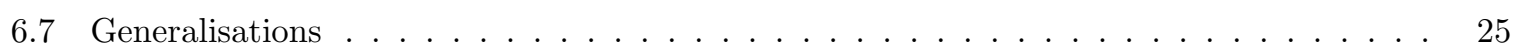

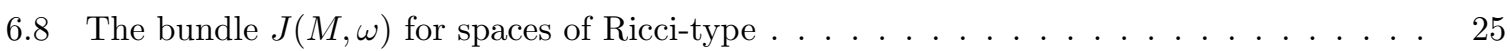

7 Ricci-flat connections $\quad 26$

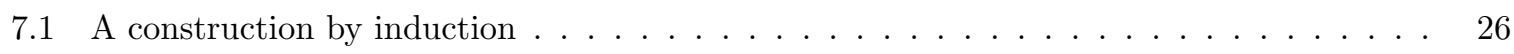

7.2 Examples of contact quadruples . . . . . . . . . . . . . . . . . . 29

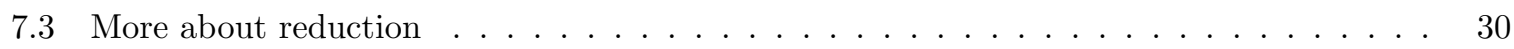

8 Non-commutative symplectic symmetric spaces 32

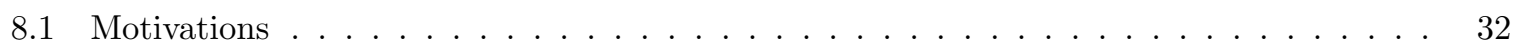

8.2 Basic definitions and the cocyclic case $\ldots \ldots \ldots \ldots \ldots \ldots \ldots \ldots \ldots$

$8.3 \quad$ A curved example: $S O(1,1) \times \mathbb{R}^{2} / \mathbb{R} \ldots \ldots \ldots \ldots \ldots \ldots \ldots \ldots$

$\begin{array}{ll}\text { References } & 39\end{array}$ 


\section{Introduction}

Symplectic geometry is by nature non-local. This is emphasized in particular by the classical Darboux theorem. The introduction on a given symplectic manifold of a symplectic connection, which is a tool adapted to local computations, may seem inappropriate. The aim of this survey is to show that symplectic geometry in the presence of special symplectic connections becomes highly rigid. More precisely there exists a family of universal models such that any symplectic manifold admitting such a special symplectic connection is locally symplectically and affinely equivalent to a particular model of the family. This local rigidity becomes a global rigidity if one requires compactness and simple connectedness.

It also appears that a twistor bundle over some of these symplectic manifolds with special connections admits a natural structure of complex analytic manifold.

Among the universal models there are certain symmetric symplectic spaces. These are particular manifolds where quantisation (whether formal or convergent) may be performed explicitly; hence they are a good framework for non-commutative geometry.

Although this survey is not exhaustive we have taken care to explain various criteria for choosing particular symplectic connections. Construction of quantisation based on a choice of symplectic connection is reduced here to one example, but we believe this example shows possibilities for development. We hope that this overview may lead others to investigate the interplay of symplectic geometry and symplectic connections.

\section{Definitions and basic facts about symplectic connections}

\subsection{Existence and non-uniqueness}

Definition 2.1 Let $(M, \omega)$ be a smooth symplectic manifold of dimension $2 n$ (i.e. $\omega$ is a closed nondegenerate 2 -form on $M)$. A symplectic connection on $(M, \omega)$ is a smooth linear connection $\nabla$ such that:

- its torsion $T^{\nabla}$ vanishes

$\left(\Leftrightarrow T^{\nabla}(X, Y):=\nabla_{X} Y-\nabla_{Y} X-[X, Y]=0\right)$;

- the symplectic form $\omega$ is parallel

$\left(\Leftrightarrow\left(\nabla_{X} \omega\right)(Y, Z):=X(\omega(Y, Z))-\omega\left(\nabla_{X} Y, Z\right)-\omega\left(Y, \nabla_{X} Z\right)=0\right)$.

To prove the existence of such a connection, take $\nabla^{0}$ any torsion free linear connection (for instance, the Levi Civita connection associated to a metric $g$ on $M$ ). Consider the tensor $N$ on $M$ defined by

$$
\nabla_{X}^{0} \omega(Y, Z)=: \omega(N(X, Y), Z) .
$$

Since $\omega$ is skewsymmetric we have $\omega(N(X, Y), Z)=-\omega(N(X, Z), Y)$ and since $\omega$ is closed we have (†) $\omega(N(X, Y), Z)=0$ where $\Varangle)$ denotes the sum over cyclic permutations of the indices $X, Y$ and ZY Define

$$
\nabla_{X} Y:=\nabla_{X}^{0} Y+\frac{1}{3} N(X, Y)+\frac{1}{3} N(Y, X)
$$


Then $\nabla$ is torsion free and:

$$
\begin{aligned}
\nabla_{X} \omega(Y, Z)= & X(\omega(Y, Z))-\omega\left(\nabla_{X} Y, Z\right)-\omega\left(Y, \nabla_{X} Z\right) \\
= & \nabla_{X}^{0} \omega(Y, Z)-\frac{1}{3} \omega(N(X, Y), Z)-\frac{1}{3} \omega(N(Y, X), Z) \\
& -\frac{1}{3} \omega(Y, N(X, Z))-\frac{1}{3} \omega(Y, N(Z, X)) \\
= & \nabla_{X}^{0} \omega(Y, Z)-\frac{1}{3} \omega(N(X, Y), Z)-\frac{1}{3} \omega(N(Y, X), Z) \\
& -\frac{1}{3} \omega(N(X, Y), Z)-\frac{1}{3} \omega(N(Z, Y), X) \\
= & \left(1-\frac{1}{3}-\frac{1}{3}\right) \omega(N(X, Y), Z)+\frac{1}{3} \omega(N(X, Z), Y)=0
\end{aligned}
$$

so the linear connection $\nabla$ is symplectic.

We shall now see how (non)-unique is a symplectic connection. Take $\nabla$ symplectic; then $\nabla_{X}^{\prime} Y:=$ $\nabla_{X} Y+S(X, Y)$ is symplectic if and only if $S(X, Y)=S(Y, X)$ (torsion free) and

$$
\begin{aligned}
0 \quad & =\nabla_{X}^{\prime} \omega(Y, Z) \\
& =\nabla_{X} \omega(Y, Z)-\omega(S(X, Y), Z)-\omega(Y, S(X, Z)) \\
& =-\omega(S(X, Y), Z)+\omega(S(X, Z), Y)
\end{aligned}
$$

hence if and only if $\omega(S(X, Y), Z)$ is totally symmetric.

Summarising we can now state the well known result:

Theorem 2.2 On a symplectic manifold $(M, \omega)$ there always exist symplectic connections. The set of symplectic connections is an affine space modelled on the space of contravariant symmetric 3-tensor fields on $M, \Gamma^{\infty}\left(S^{3} T M\right)$.

\subsection{Where do symplectic connections arise?}

The notion of symplectic connection is intimately related to that of natural formal deformation quantisation at order 2. Quantisation of a classical system is a way to pass from classical to quantum results. Deformation quantisation was introduced by Flato, Lichnerowicz and Sternheimer in [26] and in [3]; they

"suggest that quantisation be understood as a deformation of the structure of the algebra of classical observables rather than a radical change in the nature of the observables."

So deformation quantisation is defined in terms of a star product which is a formal deformation of the algebraic structure of the space of smooth functions on a symplectic (or more generally a Poisson) manifold. The associative structure given by the usual product of functions and the Lie structure given by the Poisson bracket are simultaneously deformed. Let us recall that if $(M, \omega)$ is a symplectic manifold and if $u, v \in C^{\infty}(M)$, the Poisson bracket of $u$ and $v$ is defined by

$$
\{u, v\}:=X_{u}(v)=\omega\left(X_{v}, X_{u}\right)
$$

where $X_{u}$ denotes the Hamiltonian vector field corresponding to the function $u$, i.e. such that $i\left(X_{u}\right) \omega=$ $d u$. 
Definition 2.3 A star product on a symplectic manifold $(M, \omega)$ is a bilinear map

$$
C^{\infty}(M) \times C^{\infty}(M) \rightarrow C^{\infty}(M)[[\nu]] \quad(u, v) \mapsto u *_{\nu} v:=\sum_{r \geq 0} \nu^{r} C_{r}(u, v)
$$

such that

$(u * v) * w=u *(v * w)$ (when extended $\mathbb{R}[[\nu]]$ linearly);

$C_{0}(u, v)=u v \quad C_{1}(u, v)-C_{1}(v, u)=\{u, v\} ;$

$1 * u=u * 1=u$.

If all the $C_{r}$ 's are bidifferential operators; one speaks of a differential star product; if, furthermore, each $C_{r}$ is of order $\leq r$ in each argument, one speaks of a natural star product.

The link between symplectic connections and star products appear already in the seminal paper [3] where the authors observe that if there is a flat symplectic connection $\nabla$ on $(M, \omega)$, one can generalise the classical formula for Moyal star product $*_{M}$ defined on $\mathbb{R}^{2 n}$ with a constant symplectic 2-form.

Fedosov, proved more generally that given any symplectic connection $\nabla$, one can construct a star product (in [27] it was proposed that a triple $(M, \omega, \nabla)$ be known as a Fedosov manifold):

Theorem 2.4 [25] Given a symplectic connection $\nabla$ and a sequence $\Omega=\sum_{k=1}^{\infty} \nu^{k} \omega_{k}$ of closed 2forms on a symplectic manifold $(M, \omega)$, one can build a star product $* \nabla, \Omega$ on it. This is obtained by identifying the space $C^{\infty}(M)[[\nu]]$ with a subalgebra of the algebra of sections of a bundle of associative algebras (called the Weyl bundle) on $M$. The subalgebra is the one of flat sections of the Weyl bundle, when this bundle is endowed with a flat connection whose construction is determined by the choices made of the connection on $M$ and of the sequence of closed 2-forms on $M$.

Reciprocally a natural star product determines a symplectic connection. This was first observed by Lichnerowicz [33] for a restricted class of star products.

Theorem 2.5 [28] A natural star product at order 2 determines a unique symplectic connection.

\subsection{When is there a "natural" unique symplectic connection?}

To have a canonical choice of symplectic connection on $(M, \omega)$, one needs some extra structure on the manifold.

\section{- Example 1: pseudo-Kähler manifolds}

Choose an almost complex structure $J$ on $(M, \omega)$ [i.e. $J: T M \rightarrow T M$ is a bundle endomorphism so that $\left.J^{2}=-\mathrm{Id}\right]$ so that $\left.\omega(J X, J Y)=\omega(X, Y)\right]$.

A symplectic connection $\nabla$ preserves $J$ [i.e. $\nabla J=0$ ] if and only if it is the Levi Civita connection associated to the pseudo Riemannian metric $g(X, Y):=\omega(X, J Y)$. It is thus unique and it only exists in a (pseudo-)Kähler situation.

\section{- Example 2: symmetric symplectic spaces}

Intuitively, a symmetric symplectic space is a symplectic manifold with symmetries attached to each of its points. Precisely:

Definition 2.6 A symmetric symplectic space is a triple $(M, \omega, S)$ where $(M, \omega)$ is a symplectic manifold and where $S$ is a smooth map $S: M \times M \rightarrow M$ such that, defining for any point $x \in M$ the map (called the symmetry at $x$ ):

$$
s_{x}:=S(x, \cdot): M \rightarrow M,
$$


each $s_{x}$ squares to the identity $\left[s_{x}^{2}=\mathrm{Id}\right]$ and is a symplectomorphism of $(M, \omega)\left[s_{x}^{*} \omega=\omega\right]$, $x$ is an isolated fixed point of $s_{x}$, and $s_{x} s_{y} s_{x}=s_{s_{x} y}$ for any $x, y \in M$.

Proposition 2.7 [4] On a symmetric symplectic space, there is a unique symplectic connection for which each $s_{x}$ is an affinity. It is explicitly given by

$$
\omega_{x}\left(\nabla_{X} Y, Z\right)=\frac{1}{2} X_{x} \omega\left(Y+s_{x \star} Y, Z\right) .
$$

- In the two examples above, the choice of symplectic connection was imposed by the presence of an additional structure. To select a "small" class of symplectic connections on a symplectic manifold without any additional structure, one has to choose some restrictive conditions. One way to proceed is to impose some system of equations on the curvature tensor.

\subsection{Curvature tensor of a symplectic connection}

The curvature tensor $R^{\nabla}$ of a linear connection $\nabla$ is the 2 -form on $M$ with values in the endomorphisms of the tangent bundle defined by

$$
R^{\nabla}(X, Y) Z=\left(\nabla_{X} \nabla_{Y}-\nabla_{Y} \nabla_{X}-\nabla_{[X, Y]}\right) Z
$$

for vector fields $X, Y, Z$ on $M$. If $\nabla$ is symplectic, $R_{x}^{\nabla}(X, Y)$ has values in the symplectic Lie algebra $s p\left(T_{x} M, \omega_{x}\right)=\left\{A \in \operatorname{End}\left(T_{x} M\right) \mid \omega_{x}(A u, v)+\omega_{x}(u, A v)=0, \forall u, v \in T_{x} M\right\}$.

The curvature tensor satisfies the first Bianchi identity

$$
\underset{X, Y, Z}{(\underset{7}{)}} R^{\nabla}(X, Y) Z=0
$$

where $(\Varangle)$ denotes the sum over the cyclic permutations of the listed set of elements,and the second Bianchi identity

$$
\underset{X, Y, Z}{(亠)}\left(\nabla_{X} R^{\nabla}\right)(Y, Z)=0
$$

The Ricci tensor $r^{\nabla}$ is the 2-tensor

$$
r^{\nabla}(X, Y)=\operatorname{Tr}\left(Z \mapsto R^{\nabla}(X, Z) Y\right) .
$$

The first Bianchi identity implies that $r^{\nabla}$ is symmetric.

One can define a second trace $r_{x}^{\prime}(X, Y):=\sum_{i} \omega\left(R_{x}^{\nabla}\left(e_{i}, e^{i}\right) X, Y\right)$ where the $e_{i}$ constitute a basis of $T_{x} M$ and the $e^{i}$ constitute the dual basis of $T_{x} M$ (i.e. such that $\left.\omega\left(e_{i}, e^{j}\right)=\delta_{i}^{j}\right)$. Then Bianchi's first identity implies that $r^{\prime}=-2 r^{\nabla}$.

Since the Ricci tensor is symmetric and one only has a skewsymmetric contravariant 2-tensor on $M$ (the Poisson tensor related to the symplectic form) there is no "scalar curvature".

The symplectic curvature tensor is defined as

$$
\underline{R}^{\nabla}(X, Y, Z, T):=\omega\left(R^{\nabla}(X, Y) Z, T\right) .
$$

It is antisymmetric in its first two arguments and symmetric in its last two. Hence $\underline{R}_{x}^{\nabla}$ is in $\Lambda^{2}\left(T_{x}^{*} M\right) \otimes$ $S^{2}\left(T_{x}^{*} M\right)$. To understand Bianchi's first identity, we introduce the operators of symmetrisation and skewsymmetrisation arising in the Koszul long exact sequence. 


\subsubsection{The Koszul long exact sequence}

Given any finite dimensional vector space $V$, the Koszul long exact sequence has the following form:

$$
0 \longrightarrow S^{q}(V) \stackrel{a}{\longrightarrow} V \otimes S^{q-1}(V) \stackrel{a}{\longrightarrow} \Lambda^{2} V \otimes S^{q-2}(V) \stackrel{a}{\longrightarrow} \cdots \stackrel{a}{\longrightarrow} \Lambda^{q-1}(V) \otimes V \stackrel{a}{\longrightarrow} \Lambda^{q}(V) \longrightarrow 0
$$

where $a$ is the skewsymmetrisation operator:

$$
a\left(v^{1} \wedge \ldots \wedge v^{q} \otimes w^{1} \cdots w^{p}\right)=\sum_{i=1}^{p} v^{1} \wedge \ldots \wedge v^{q} \wedge w^{i} \otimes w^{1} \cdots w^{i-1} w^{i+1} \cdots w^{p} .
$$

The symmetrisation operator reads:

$$
s\left(v^{1} \wedge \ldots \wedge v^{q} \otimes w^{1} \ldots w^{p}\right) \sum_{i=1}^{q}(-1)^{q-i} v^{1} \wedge \ldots v^{i-1} \wedge v^{i+1} \ldots \wedge v^{q} \otimes v^{i} \cdot w^{1} \ldots w^{p} .
$$

These two operators satisfy $a^{2}=0, s^{2}=0,\left(a_{\circ} s+s_{\circ} a\right)_{\left.\right|_{\Lambda^{q} V \otimes S^{p}(V)}}=(p+q)$ Id .

The first Bianchi identity on the value at a point $x$ of the symplectic curvature tensor takes the form:

$$
\underset{X, Y, Z}{\stackrel{(}{(})} \underline{R}_{x}^{\nabla}(X, Y, Z, T)=0 \Leftrightarrow \underline{R}_{x}^{\nabla} \in \operatorname{ker} a \subset \Lambda^{2}\left(T_{x}^{*} M\right) \otimes S^{2}\left(T_{x}^{*} M\right) .
$$

The space $\mathscr{R}_{x}$ of 4 -tensors satisfying the algebraic identities of a symplectic curvature tensor at $x$ is:

$$
\underline{\mathscr{R}}_{x}:=\operatorname{ker} a_{\left.\right|_{\Lambda^{2}(V) \otimes S^{2}(V)}} \simeq\left(V \otimes S^{3}(V)\right) / S^{4}(V) \quad \text { for } V=T_{x}^{*} M .
$$

\subsubsection{Decomposition of the curvature}

The group $S p\left(T_{x} M, \omega_{x}\right)=\left\{A \in \operatorname{End}\left(T_{x} M\right) \mid \omega_{x}(A u, A v)=\omega_{x}(u, v) \forall u, v \in T_{x} M\right\}$ acts on $V=T_{x}^{*} M$ and thus on $\underline{\mathscr{R}}_{x} \simeq\left(V \otimes S^{3}(V)\right) / S^{4}(V)$. Under this action the space $V \otimes S^{3}(V)$, in dimension $2 n \geq 4$, decomposes into three irreducible subspaces $\left(S^{4}(V) \oplus S^{\prime 2}(V) \oplus W\right.$ where $S^{\prime 2}(V)=a\left(s\left(\omega_{x} \otimes S^{2}(V)\right)\right) \sim$ $S^{2}(V)$ so that:

$$
\underline{\mathscr{R}}_{x}=\underline{\mathscr{E}}_{x} \oplus \underline{\mathscr{W}}_{x} .
$$

The decomposition of the symplectic curvature tensor $\underline{R}_{x}^{\nabla}$ into its $\underline{\mathscr{E}}_{x}$ component (denoted $\underline{E}_{x}^{\nabla}$ ) and its $\underline{W}_{x}$ component (denoted $\left.\underline{W}_{x}^{\nabla}\right)$,

$$
\underline{R}_{x}^{\nabla}=\underline{E}_{x}^{\nabla}+\underline{W}_{x}^{\nabla}
$$

is given by

$$
\begin{aligned}
\underline{E}_{x}^{\nabla}(X, Y, Z, T)= & -\frac{1}{2(n+1)}\left[2 \omega_{x}(X, Y) r_{x}^{\nabla}(Z, T)+\omega_{x}(X, Z) r_{x}^{\nabla}(Y, T)\right. \\
& \left.+\omega_{x}(X, T) r_{x}^{\nabla}(Y, Z)-\omega_{x}(Y, Z) r_{x}^{\nabla}(X, T)-\omega_{x}(Y, T) r_{x}^{\nabla}(X, Z)\right] .
\end{aligned}
$$

The corresponding decomposition of the curvature tensor (see Vaisman [45]) has the form

$$
R_{x}^{\nabla}=E_{x}^{\nabla}+W_{x}^{\nabla}
$$

where

$$
\begin{gathered}
E^{\nabla}(X, Y) Z=\frac{1}{2 n+2}\left(2 \omega(X, Y) \rho^{\nabla} Z+\omega(X, Z) \rho^{\nabla} Y-\omega(Y, Z) \rho^{\nabla} X\right. \\
\left.+\omega\left(X, \rho^{\nabla} Z\right) Y-\omega\left(Y, \rho^{\nabla} Z\right) X\right)
\end{gathered}
$$


with $r^{\nabla}$ converted into an endomorphism $\rho^{\nabla}$ given by

$$
\omega\left(X, \rho^{\nabla} Y\right)=r^{\nabla}(X, Y) .
$$

Definition 2.8 A symplectic connection $\nabla$ on $(M, \omega)$ will be said to be of Ricci-type if $W^{\nabla}=0$; it will be said to be Ricci-flat if $E^{\nabla}=0$ (hence if and only if $r^{\nabla}=0$ ).

One can combine restricting the holonomy algebra $\mathfrak{g} \subset s p\left(\mathbb{R}^{2 n}, \Omega\right)$ and the vanishing of some components of the curvature when the curvature is decomposed into irreducible components under the action of $\mathfrak{g}$ to define special symplectic connections; these will appear in section 5 .

\subsection{Variational principle}

To select symplectic connections through a variational principle [13], one can consider a Lagrangian $L\left(R^{\nabla}\right)$, which is a polynomial in the curvature of the connection $\nabla$, invariant under the action of the symplectic group

$$
\int_{M} L\left(R^{\nabla}\right) \omega^{n}
$$

From what we have seen before, there is no invariant polynomial of degree 1 in the curvature, so the easiest choice is a polynomial of degree 2 in $R^{\nabla}$. The space of degree 2 polynomials in the curvature which are invariant under the action of the symplectic group is 2-dimensional and spanned by $E^{\nabla} \cdot E^{\nabla}$ and $W^{\nabla} \cdot W^{\nabla}$ (or, equivalently by $R^{\nabla} \cdot R^{\nabla}$ and $r^{\nabla} \cdot r^{\nabla}$ ) where $\cdot$ denotes the symmetric function-valued product of tensors induced by $\omega$ and $S \cdot T$, for $S$ and $T$ tensor-fields on $M$ of the same type, is given in local coordinates by

$$
S \cdot T=\left(\omega^{-1}\right)^{i_{1} i_{1}^{\prime}} \cdots\left(\omega^{-1}\right)^{i_{p} i_{p}^{\prime}} \omega_{j_{1} j_{1}^{\prime}} \cdots \omega_{j_{q} j_{q}^{\prime}} S_{i_{1} \ldots i_{p}}^{j_{1} \ldots j_{q}} T_{i_{1}^{\prime} \ldots j_{p}^{\prime}}^{j_{1}^{\prime} \ldots j_{q}^{\prime}}
$$

From Chern-Weil theory we know that the first Pontryagin class is represented by a 4 -form $P_{1}(\nabla)$ which is built from an invariant combination of the curvature and the symplectic form. In fact

$$
P_{1}(\nabla) \wedge \omega^{n-2}=\frac{1}{16 \pi^{2}}\left[r^{\nabla} \cdot r^{\nabla}-\frac{1}{2} R^{\nabla} \cdot R^{\nabla}\right] \omega^{n}
$$

Since this combination will be constant under variations, all non-trivial Euler equations coming from a variational principle built from a second order invariant polynomial in the curvature are the same and take the form:

$$
\underset{X, Y, Z}{(亠}\left(\nabla_{X} r^{\nabla}\right)(Y, Z)=0
$$

Definition 2.9 A symplectic connection $\nabla$ is said to be preferred if it is a solution of Equation (7).

\section{Preferred symplectic connections}

The preferred symplectic connections $\nabla$ on a symplectic manifold $(M, \omega)$ of dimension $2 n$ are critical points of the functional

$$
\int_{M} \operatorname{Tr}\left(\rho^{\nabla}\right)^{2} \frac{\omega^{n}}{n !}
$$


where $\rho^{\nabla}$ is the Ricci endomorphism as previously defined in (6). They obey the system of second order partial differential equations

$$
\underset{X, Y, Z}{\stackrel{\Psi}{\Psi}}\left(\nabla_{X} r^{\nabla}\right)(Y, Z)=0
$$

where $r^{\nabla}$ is the Ricci tensor of the connection $\nabla$.

The basic problem is to determine if on a given manifold $(M, \omega)$ there exists a preferred connection and "how many" different ones may occur; two solutions are different in this context if they are not related through a symplectic diffeomorphism.

This basic problem is essentially solved when $(M, \omega)$ is a compact orientable surface. We give also a partial answer for the standard symplectic plane $\left(\mathbb{R}^{2}, \Omega_{0}\right)$.

When the dimension of $M$ is $\geq 4$, we can give large classes of examples of preferred connections:

(i) examples of symplectic manifolds admitting a Ricci-flat connection;

(ii) examples of symplectic manifolds admitting a "Ricci-parallel" connection;

(iii) examples of homogeneous symplectic manifolds admitting a homogeneous preferred symplectic connection.

\subsection{Preferred symplectic connections in dimension 2}

The study of preferred connections in dimension 2 relies on a function $\beta$, depending on the connection, which first appears in

Lemma 3.1 [13] Let $(M, \omega)$ be a symplectic surface and let $\nabla$ be a preferred symplectic connection on it. Then

(i) there exists a 1-form u such that, for any vector fields $X, Y, Z$ one has

$$
\left(\nabla_{X} r^{\nabla}\right)(Y, Z)=\omega(Y, X) u(Z)+\omega(Z, X) u(Y)
$$

(ii) there exists a function $\beta$ such that

$$
\nabla_{X} u=\beta \omega
$$

(iii) defining the vector field $\bar{u}$ by

$$
i(\bar{u}) \omega=u,
$$

one has, for any vector fields $X, Y$ :

$$
\begin{aligned}
X \beta & =-r^{\nabla}(X, \bar{u}), \\
\left(\nabla^{2} \beta\right)(X, Y) & =-u(X) u(Y)+\beta r^{\nabla}(X, Y) ;
\end{aligned}
$$

(iv) there exist two real numbers $A$ and $B$ such that

$$
\begin{aligned}
r^{\nabla}(\bar{u}, \bar{u}) & =\beta^{2}+B, \\
\frac{1}{4} \operatorname{Tr}\left(\rho^{\nabla}\right)^{2} & =\beta+A .
\end{aligned}
$$

Hence $\nabla$ is locally symmetric (i.e. $\nabla R^{\nabla}=0$ ) if and only if $\beta=0$. If $M$ is compact, the second property above shows that $\beta$ can not be a non-vanishing constant (it would indeed imply that the symplectic 2 -form $\omega$ be exact). If $\beta$ is not a constant and $M$ is compact, a detailed study of the critical 
points of $\beta$ permits to show that no compact symplectic surface of positive genus admits a non-locally symmetric preferred symplectic connection. The case of the 2-sphere is more delicate and requires precise estimates. The conclusion is

Theorem 3.2 [13] A preferred connection on a compact symplectic surface is necessarily locally symmetric.

Globally symmetric symplectic surfaces may be completely described. Locally symmetric symplectic surfaces may be simply related to the globally symmetric ones provided the connection is geodesically complete. A case by case analysis leads to

Theorem 3.3 [13, 4] Let $(M, \omega, \nabla)$ be a compact symplectic surface endowed with a complete locally symmetric symplectic connection. Then, up to diffeomorphisms, either

- $M$ is the 2-sphere $S^{2}$ with $\omega$ a multiple of the standard volume form and with $\nabla$ the Levi Civita connection of the standard Riemannian metric with constant positive curvature equal to 1 ;

- $M$ is the torus $T^{2}$ with $\omega$ a multiple of the standard invariant volume form and with $\nabla$ a flat affine symplectic connection;

- $M$ is a surface $\Sigma_{g}$ of genus $g \geq 2$ with $\nabla$ a multiple of the standard volume form inherited from the disk and with $\nabla$ the connection associated to a metric $h$ of constant negative curvature equal to -1 .

What can be said about the existence and the number of different complete preferred connections on a given compact symplectic surface?

Consider the case of $S^{2}$ with a symplectic structure $\omega$. There exists a positive real number $k$ so that $\int_{S^{2}} \omega=k \int_{S^{2}} \omega_{0}$ where $\omega_{0}$ is the standard symplectic structure on $S^{2}$ defining the same orientation as $\omega$. Thus the de Rham cohomology classes defined by $\omega$ and $k \omega_{0}$ are the same. Furthermore $(1-t) \omega+t k \omega_{0}$ is symplectic for any $t \in[0,1]$ and defines the same cohomology class. By Moser's stability theorem (see [34]), there exists a 1-parametric family $\varphi_{t}$ of diffeomorphisms of the sphere $S^{2}$ such that $\varphi_{0}=$ id and $\varphi_{1}^{*} \omega=k \omega_{0}$. If $g_{0}$ is the standard metric on $S^{2}$, define the metric $g$ on the sphere to be such that $\varphi_{1}^{*} g=k g_{0}$. The Levi Civita connection associated to $g$ is clearly symplectic relative to $\omega$ and symmetric, hence preferred. Furthermore, if $\nabla^{\prime}$ is another symplectic connection on $\left(S^{2}, \omega\right)$ which is preferred and complete, it is automatically symmetric since $S^{2}$ is simply connected and it coincides with $\nabla$. Thus

Theorem 3.4 [13] On $\left(S^{2}, \omega\right)$ there exists a complete symplectic preferred connection and any complete preferred connection is the image of that one through a symplectomorphism.

The result on the existence is obtained similarly on the torus $T^{2}$ or the surface $\Sigma_{g}$ endowed with any symplectic structure. In these cases, there is no unicity (because of the choice of the affine connection or the choice of the metric).

The non-compact situation is more complicated. One can show that on the plane endowed with the standard constant symplectic structure $\left(\mathbb{R}^{2}, \Omega_{0}\right)$, there exist five affinely distinct globally symmetric complete symplectic connections. There also exist a 2-parametric family of non-homogeneous preferred symplectic connections which are not locally symmetric. 


\subsection{Ricci-flat connections}

A symplectic connection is said to be Ricci-flat if its Ricci tensor $r^{\nabla}$ vanishes. Obviously, those give examples of preferred connections! We explain in Section 7 a construction of Ricci-flat connections: when a symplectic manifold $(M, \omega)$ of dimension $2 n \geq 4$ is the first element of a contact quadruple, any symplectic connection $\nabla$ on $(M, \omega)$ can be lifted to define a Ricci-flat symplectic connection on a certain symplectic manifold $\left(P, \omega^{\prime}\right)$ of dimension $2 n+2$. This procedure gives examples of Ricci-flat, non-flat, symplectic connections in any dimension $\geq 6$.

\subsection{Ricci-parallel symplectic connections}

Let $(M, \omega, \nabla)$ be a symplectic manifold with a symplectic connection; assume there exists on this manifold a compatible almost complex structure $J$ (i.e. at each point $x$ in $M, J_{x} \in \operatorname{End}\left(T_{x} M\right), J_{x}^{2}=$ - Id,$\omega(J X, J Y)=\omega(X, Y)$ ) which is parallel (i.e. $\nabla J=0)$. Then the pseudo-riemannian metric $g$ defined by $g(X, Y):=\omega(X, J Y)$ is also parallel, so the connection is the Levi Civita connection for $g$ and the manifold is pseudo-Kähler. If this connection is preferred one has:

Theorem 3.5 [19] Let $(M, \omega, J)$ be a pseudo-Kähler manifold. If the Levi Civita connection is preferred, then the Ricci tensor is parallel.

This gives examples of Ricci-parallel symplectic connections.

Consider now a symplectic manifold $(M, \omega)$ endowed with a Ricci-parallel symplectic connection $\nabla$. Write

$$
T_{x} M^{\mathbb{C}}=\oplus_{\lambda \in \operatorname{spec}}\left(T_{x} M^{\mathbb{C}}\right)_{\lambda}
$$

where $\left(T_{x} M^{\mathbb{C}}\right)_{\lambda}$ is the generalised eigenspace for $\rho_{x}^{\nabla}$,i.e.

$$
\left(T_{x} M^{\mathbb{C}}\right)_{\lambda}=\left\{X \in T_{x} M^{\mathbb{C}} \mid\left(\rho_{x}^{\nabla}-\lambda\right)^{2 n} X=0\right\}
$$

and spec denotes the set of different eigenvalues of $\rho_{x}^{\nabla}$ on $T_{x} M^{\mathbb{C}}$.

Observe that $\left(T_{x} M^{\mathbb{C}}\right)_{\lambda}$ and $\left(T_{x} M^{\mathbb{C}}\right)_{\mu}$ are orthogonal with respect to the symplectic form $\omega_{x}$ unless $\mu=-\lambda$. We define real symplectic subspaces of $T_{x} M$ :

$R_{\lambda}$, for each real positive eigenvalue $\lambda$, so that the complexification of $R_{\lambda}$ is given by $R_{\lambda}^{\mathbb{C}}=\left(T_{x} M^{\mathbb{C}}\right)_{\lambda} \oplus$ $\left(T_{x} M^{\mathbb{C}}\right)_{-\lambda}$; we denote by $R$ the set of such eigenvalues;

$I_{\lambda}$ for each purely imaginary eigenvalue $\lambda=i a, a>0$, so that its complexification is $I_{\lambda}^{\mathbb{C}}=\left(T_{x} M^{\mathbb{C}}\right)_{\lambda} \oplus$ $\left(T_{x} M^{\mathbb{C}}\right)_{-\lambda}$; we denote by $I$ the set of such eigenvalues;

$C_{\lambda}$ for each eigenvalue $\lambda=a+i b, a>0, b>0$, so that its complexification is $C_{\lambda}^{\mathbb{C}}=\left(T_{x} M^{\mathbb{C}}\right)_{\lambda} \oplus$ $\left(T_{x} M^{\mathbb{C}}\right)_{-\lambda} \oplus\left(T_{x} M^{\mathbb{C}}\right)_{\bar{\lambda}} \oplus\left(T_{x} M^{\mathbb{C}}\right)_{-\bar{\lambda}}$; we denote by $C$ the set of such eigenvalues.

They give a symplectic orthogonal decomposition of $T_{x} M$ :

$$
T_{x} M=T_{x} M_{0} \oplus\left(\oplus_{\lambda \in R} R_{\lambda}\right) \oplus\left(\oplus_{\lambda \in I} I_{\lambda}\right) \oplus\left(\oplus_{\lambda \in C} C_{\lambda}\right) .
$$

Ricci-parallel implies that the Ricci endomorphism $\rho^{\nabla}$ commutes with all curvature endomorphisms so that all distributions corresponding to the above defined subspaces are parallel. We get

Theorem 3.6 [19, 16] Let $(M, \omega)$ be a $2 n$-dimensional symplectic manifold endowed with a symplectic connection $\nabla$ whose Ricci tensor is parallel. Assume that the Ricci tensor is not degenerate. Then 
1 the connection $\nabla$ is the Levi Civita connection associated to the metric defined by the Ricci tensor $r^{\nabla}$;

2 the distributions $R_{\lambda}, I_{\lambda}, C_{\lambda}$ are parallel, symplectic, and $\rho^{\nabla}$ restricted to any of these is semisimple;

3 if $M$ is simply connected and $\nabla$ complete, then $M$ is symplectomorphic and affinely equivalent to the product of the symplectic submanifolds corresponding to the integral leaves of the distributions $R_{\lambda}, I_{\lambda}, C_{\lambda}$;

4 the manifolds corresponding to the leaves of $R_{\lambda}$ admit two parallel, transverse Lagrangian foliations; those corresponding to $I_{\lambda}$ are Kähler-Einstein manifolds; those corresponding to $C_{\lambda}$ have dimension $4 k$ if $k$ is the multiplicity of $\lambda$ and also admit two parallel transverse Lagrangian foliations;

5 if all the eigenvalues of $\rho^{\nabla}$ have multiplicity one, the factors are two dimensional or four dimensional symplectic symmetric spaces.

\subsection{Homogeneous preferred connections}

If $(M, \omega)$ is a connected, simply connected, compact, homogeneous, symplectic manifold, a classical result of Kostant tells us that $(M, \omega)$ is symplectomorphic to a coadjoint orbit of a compact semi simple Lie group $G$, endowed with its standard Lie-Kirillov-Kostant-Souriau symplectic structure.

Let $\nabla$ be a symplectic connection on $(M, \omega)$ stable by the action of $G$. Let $p \in M$ and let $\pi$ : $G \rightarrow M g \mapsto g . p$ be the canonical projection related to the choice of the base point $p$. Let $H$ be the stabilizer of $p$ in $G(H:=\{g \in G, \mid g \cdot p=p\})$ and let $\mathfrak{g}$ (resp. $\mathfrak{h}$ ) be the Lie algebra of $G$ (resp. $H)$. For any element $X \in \mathfrak{g}$, let $X^{*}$ denotes the fundamental vector field on $M$ associated to $X$, i.e. $X_{x}^{*}=\frac{d}{\left.d t\right|_{0}} \exp -t X . x$. Write $\mathfrak{g}=\mathfrak{h} \oplus \mathfrak{m}$ where $\mathfrak{m}$ is the orthogonal to $\mathfrak{h}$ relative to the Killing form. Each tangent vector at $p$ is the value at $p$ of a (unique) fundamental vector field $Y^{*}$ with $Y \in \mathfrak{m}$. Define a $\operatorname{map} D: \mathfrak{m} \rightarrow \operatorname{End}(\mathfrak{m})$ by

$$
\left(\nabla_{X^{*}} Y^{*}\right)_{p}=(D(X) Y)_{p}^{*}
$$

This map $D$ entirely determines the $G$-invariant connection $\nabla$. It satisfies two conditions:

$$
\begin{aligned}
D(X) Y-D(Y) X-\pi_{\mathfrak{m}}([X, Y]) & =0 \\
\Omega(D(Y) X, Z)+\Omega(Y, D(Z) X) & =0
\end{aligned}
$$

where $\pi_{\mathfrak{m}}$ is the projection of $\mathfrak{g}$ on $\mathfrak{m}$ relatively to the decomposition $\mathfrak{g}=\mathfrak{h} \oplus \mathfrak{m}$, and where $\Omega=$ $\left(\pi^{*} \omega_{p}\right)_{\mid \mathfrak{m} \times \mathfrak{m}}$. The space of invariant homogeneous symplectic connections on $M$ may be identified to the space of maps $D: \mathfrak{m} \rightarrow \operatorname{End}(\mathfrak{m})$ satisfying the conditions 10 . Such a connection is preferred if it is a critical point of the functional

$$
\mathscr{I}(\nabla)=\int_{M} \operatorname{Tr}\left(\rho^{\nabla}\right)^{2} \frac{\omega^{n}}{n !} .
$$

By Palais's principle, to determine the $G$-invariant critical points of $\mathscr{I}$, it is sufficient to determine the critical points of the restriction of $\mathscr{I}$ to the space of $G$-invariant connections [37]. For an invariant connection, $\mathscr{I}$ reduces to $\operatorname{Tr}\left(\rho^{\nabla}\right)^{2} \operatorname{Vol}(M)$ and one shows that the Ricci tensor is a polynomial of degree 2 on $D$.

Lemma 3.7 [18] The functional $\mathscr{I}$ is a fourth order polynomial on $D$. 
Using the structure of semisimple algebras of compact type, one shows that this polynomial is nonnegative and that the homogeneous polynomial corresponding to the terms of degree 4 is strictly positive outside the origin. In fact one replaces $D$ by $D^{\prime}=D-\frac{1}{2} \pi_{\mathfrak{m}} \circ$ ad; the conditions 10 simply express the fact that the 3 -form $\Omega\left(D^{\prime}(\cdot) \cdot, \cdot\right)$ is completely symmetric.

Lemma 3.8 [18] If $P: \mathbb{C}^{N} \rightarrow \mathbb{R}$ is a non-negative, real valued polynomial of order $d$, such that the homogeneous terms of order $d$ are strictly positive outside the origin, then $P$ has a minimum.

From lemmas 3.7 and 3.8 one gets:

Theorem 3.9 [18] Every coadjoint orbit of a compact semi-simple Lie group $G$ admits a preferred invariant symplectic connection.

In the case of a coadjoint orbit of $S U(3)$, one can prove by direct calculation that this preferred connection is unique.

\section{Ricci-type connections}

Ricci-type connections were defined in Definition 2.8; they are symplectic connections whose curvature tensor is entirely determined by the Ricci tensor, i.e. for which $W^{\nabla}=0$.

\subsection{Some properties of the curvature of a Ricci-type connection}

Let $(M, \omega)$ be a smooth symplectic manifold of $\operatorname{dim} 2 n(n \geq 2)$ and let $\nabla$ be a smooth Ricci-type symplectic connection. The following results follow directly from the definition (and Bianchi's second identity).

Lemma 4.1 [20] The curvature endomorphism has the form

$$
R^{\nabla}(X, Y)=-\frac{1}{2(n+1)}\left[-2 \omega(X, Y) \rho^{\nabla}-\rho^{\nabla} Y \otimes \underline{X}+\rho^{\nabla} X \otimes \underline{Y}-X \otimes \underline{\rho^{\nabla} Y}+Y \otimes \underline{\rho^{\nabla} X}\right]
$$

where $\underline{X}$ denotes the 1-form $i(X) \omega$ (for $X$ a vector field on $M$ ) and where, as before, $\rho^{\nabla}$ is the endomorphism associated to the Ricci tensor by $r^{\nabla}(U, V)=\omega\left(U, \rho^{\nabla} V\right)$.

Furthermore:

(i) there exists a vector field $U^{\nabla}$ such that

$$
\nabla_{X} \rho^{\nabla}=-\frac{1}{2 n+1}\left[X \otimes \underline{U}^{\nabla}+U^{\nabla} \otimes \underline{X}\right]
$$

(ii) there exists a function $f^{\nabla}$ such that

$$
\nabla_{X} U^{\nabla}=-\frac{2 n+1}{2(n+1)}\left(\rho^{\nabla}\right)^{2} X+f^{\nabla} X
$$

(iii) there exists a real number $K^{\nabla}$ such that

$$
\operatorname{tr}\left(\rho^{\nabla}\right)^{2}+\frac{4(n+1)}{2 n+1} f^{\nabla}=K^{\nabla} .
$$

Corollary 4.2 Any Ricci-type symplectic connection is preferred

The fact that $\underset{X Y Z}{\hookrightarrow} \nabla_{X} r \nabla(Y, Z)=0$ follows immediately from (12). 


\subsection{A construction by reduction}

Consider the manifold $M=\mathbb{R}^{2 n+2}$ with its standard symplectic structure $\Omega^{\prime}$.

Let $A$ be a non-zero element in the symplectic Lie algebra $s p\left(\mathbb{R}^{2 n+2}, \Omega^{\prime}\right)$.

Let $\Sigma_{A}$ be the closed hypersurface $\Sigma_{A} \subset \mathbb{R}^{2 n+2}$ defined by

$$
\Sigma_{A}=\left\{x \in \mathbb{R}^{2 n+2} \mid \Omega^{\prime}(x, A x)=1\right\} .
$$

(In order for $\Sigma_{A}$ to be non-empty we replace, if necessary, $A$, by $-A$.)

The 1-parameter subgroup $\exp t A$ of the symplectic group acts on $\mathbb{R}^{2 n+2}$, preserving $\Omega^{\prime}$ and $\Sigma_{A}$; [the corresponding fundamental vector field $A^{*}$ on $\mathbb{R}^{2 n+2}$ (defined by $A_{x}^{*}:=\frac{d}{d t} \exp -t A x_{\left.\right|_{0}}=-A x$ ) is Hamiltonian, i.e. $i\left(A^{*}\right) \omega=d H_{A}$, with $H_{A}(x)=\frac{1}{2} \Omega(x, A x)$ and $\Sigma_{A}$ is a level set of this Hamiltonian].

We shall consider the reduced space $M^{r e d}:=\Sigma_{A} /\{\exp t A \mid t \in \mathbb{R}\}$ with the canonical projection $\pi: \Sigma_{A} \rightarrow M^{\text {red }}$. This can always be locally defined as follows.

Since the vector field $A x$ is nowhere 0 on $\Sigma_{A}$, for any $x_{0} \in \Sigma_{A}$, there exist:

- a neighbourhood $U_{x_{0}}\left(\subset \Sigma_{A}\right)$,

- a ball $D^{\text {red }} \subset \mathbb{R}^{2 n}$ of radius $r_{0}$, centred at the origin,

- a real interval $I=(-\epsilon, \epsilon)$

- and a diffeomorphism

$$
\chi: D^{r e d} \times I \rightarrow U_{x_{0}}
$$

such that $\chi(0,0)=x_{0}$ and $\chi(y, t)=\exp -t A(\chi(y, 0))$. We shall denote

$$
\pi: U_{x_{0}} \rightarrow D^{r e d} \quad \pi=p_{1} \otimes \chi^{-1}
$$

The space $D^{\text {red }}$ is a local version of the Marsden-Weinstein reduction of $\Sigma_{A}$ around the point $x_{0}$.

If $\left.x \in \Sigma_{A}, T_{x} \Sigma_{A}=\right\rangle A x\left\langle^{\perp}\right.$, where $\rangle v_{1}, \ldots, v_{p}\left\langle\right.$ denotes the subspace spanned by $v_{1}, \ldots, v_{p}$ and $\perp$ denotes the orthogonal relative to $\Omega^{\prime}$; let $\left.\mathscr{H}_{x}\left(\subset T_{x} \Sigma_{A}\right)=\right\rangle x, A x\left\langle^{\perp}\right.$; then $\pi_{* x}$ defines an isomorphism between $\mathscr{H}_{x}$ and the tangent space $T_{y} D^{\text {red }}$ for $y=\pi(x)$.

A reduced symplectic form on $D^{\text {red }}, \omega^{\text {red }}$, is defined by

$$
\omega_{y=\pi(x)}^{r e d}(X, Y):=\Omega_{x}^{\prime}\left(\bar{X}_{x}, \bar{Y}_{x}\right)
$$

where $\bar{Z}$ denotes the horizontal lift of $Z \in T_{y} D^{\text {red }}$; i.e. $\bar{Z} \in \mathscr{H}_{x}$ and $\pi_{* x}(\bar{Z})=Z$.

Let $\nabla$ be the standard flat symplectic affine connection on $\mathbb{R}^{2 n+2}$. The reduced symplectic connection $\nabla^{\text {red }}$ on $D^{\text {red }}$ is defined by

$$
\left(\nabla_{X}^{r e d} Y\right)_{y}:=\pi_{*_{x}}\left(\nabla_{\bar{X}} \bar{Y}-\Omega^{\prime}(A \bar{X}, \bar{Y}) x+\Omega^{\prime}(\bar{X}, \bar{Y}) A x\right)
$$

Proposition 4.3 [2] The manifold $\left(D^{\text {red }}, \omega^{\text {red }}\right)$ is a symplectic manifold and $\nabla^{\text {red }}$ is a symplectic connection of Ricci-type on it.

Furthermore, a direct computation shows that the corresponding $\rho^{\nabla^{\text {red }}}, U^{\nabla^{\text {red }}}$ and $f^{\nabla^{\text {red }}}$ are given by:

$$
\begin{aligned}
\overline{\rho^{\nabla^{r e d}} X}(x) & =-2(n+1) \overline{A_{x}} \bar{X} \\
\bar{U}^{\nabla^{r e d}}(x) & =-2(n+1)(2 n+1) \overline{A_{x}^{2}} x \\
\left(\pi^{*} f^{\nabla^{r e d}}\right)(x) & =2(n+1)(2 n+1) \Omega^{\prime}\left(A^{2} x, A x\right)
\end{aligned}
$$


where $\overline{A_{x}^{k}}$ is the map induced by $A^{k}$ with values in $\mathscr{H}_{x}$ :

$$
\overline{A_{x}^{k}}(X)=A^{k} X+\Omega^{\prime}\left(A^{k} X, x\right) A x-\Omega^{\prime}\left(A^{k} X, A x\right) x .
$$

\subsection{Local models for Ricci-type connections}

We have seen that given a Ricci-type symplectic connection $\nabla$ on a symplectic manifold $(M, \omega)$ the curvature $R^{\nabla}$ is entirely determined by $\rho^{\nabla}$ (11); its covariant derivative $\nabla R^{\nabla}$ is thus determined by $\nabla \rho^{\nabla}$ which in turn is determined by the vector field $U^{\nabla}(12)$. The second covariant derivative $\nabla^{2} R^{\nabla}$ is determined by $\nabla U^{\nabla}$ hence by $\rho^{\nabla}$ and $f^{\nabla}$ (13). Since $f^{\nabla}$ satisfies equation (14), all successive covariant derivatives of the curvature tensor are determined by $\rho^{\nabla}, U^{\nabla}$ and $K^{\nabla}$.

Corollary 4.4 Let $(M, \omega)$ be a smooth symplectic manifold of dimension $2 n(n \geq 2)$ and let $\nabla$ be a smooth Ricci-type connection. Let $p_{0} \in M$; then the curvature $R_{p_{0}}^{\nabla}$ and its covariant derivatives $\left(\nabla^{k} R^{\nabla}\right)_{p_{0}}($ for all $k)$ are determined by $\left(\rho_{x_{0}}^{\nabla}, U_{x_{0}}^{\nabla}, K^{\nabla}\right)$.

Corollary 4.5 Let $(M, \omega, \nabla)$ (resp. $\left.\left(M^{\prime}, \omega^{\prime}, \nabla^{\prime}\right)\right)$ be two symplectic manifolds of the same dimension $2 n(n \geq 2)$ each of them endowed with a symplectic connection of Ricci-type. Assume that there exists a linear map $b: T_{x_{0}} M \rightarrow T_{x_{0}^{\prime}} M^{\prime}$ such that

(i) $b^{*} \omega_{x_{0}^{\prime}}^{\prime}=\omega_{x_{0}}$,

(ii) $b u_{x_{0}}^{\nabla}=u_{x_{0}^{\prime}}^{\nabla}$,

(iii) $b \circ \rho_{x_{0}}^{\nabla} \circ b^{-1}=\rho_{x_{0}^{\prime}}^{\nabla^{\prime}}$.

Assume further that $K^{\nabla}=K^{\nabla^{\prime}}$. Then the manifolds are locally affinely symplectically isomorphic, i.e. there exists a normal neighbourhood of $x_{0}$ (resp. $\left.x_{0}^{\prime}\right) U_{x_{0}}$ (resp. $\left.U_{x_{0}^{\prime}}^{\prime}\right)$ and a symplectic affine diffeomorphism $\varphi:\left(U_{x_{0}}, \omega, \nabla\right) \rightarrow\left(U_{x_{0}^{\prime}}^{\prime}, \omega^{\prime}, \nabla^{\prime}\right)$ such that $\varphi\left(x_{0}\right)=x_{0}^{\prime}$ and $\varphi_{* x_{0}}=b$.

In case the symplectic manifold and the connection are real analytic, this follows from classical results, see for instance Theorem 7.2 and Corollary 7.3 in Kobayashi-Nomizu Volume 1 [31]. However, connections of Ricci-type are always real analytic, as we shall see in Section 5.

Theorem 4.6 Any symplectic manifold with a Ricci-type connection is locally symplectically affinely isomorphic to the symplectic manifold with a Ricci-type connection obtained by a local reduction procedure around $e_{0}=(1,0, \ldots, 0)$ from a constraint surface $\Sigma_{A}$ defined by a second order polynomial $H_{A}$ for $A \in \operatorname{sp}\left(\mathbb{R}^{2 n+2}, \Omega^{\prime}\right)$ in the standard symplectic manifold $\left(\mathbb{R}^{2 n+2}, \Omega^{\prime}\right)$ endowed with the standard flat connection.

Indeed if $p \in M$ and if $\xi$ is a symplectic frame at $p$ [i.e. $\xi:\left(\mathbb{R}^{2 n}, \Omega^{(2 n)}\right) \rightarrow\left(T_{p}, \omega_{p}\right)$ is a symplectic isomorphism of vector spaces], one defines

$$
\tilde{u}(\xi)=(\xi)^{-1} U^{\nabla}(p), \quad \tilde{\rho}(\xi)=(\xi)^{-1} \rho^{\nabla}(p) \xi
$$

and

$$
\tilde{A}(\xi)=\left(\begin{array}{ccc}
0 & \frac{f(p)}{2(n+1)(2 n+1)} & \frac{-\tilde{u}(\xi)}{2(n+1)(2 n+1)} \\
1 & 0 & 0 \\
0 & \frac{-\tilde{u}(\xi)}{2(n+1)(2 n+1)} & \frac{-\tilde{\rho}(\xi)}{2(n+1)}
\end{array}\right)
$$


with $\underline{u}:=\Omega^{\prime}(u, \cdot)$ and one looks at the reduction for this $A=\tilde{A}(\xi)$.

\subsection{Global models for Ricci-type connections}

Theorem 4.7 [21] If $(M, \omega, \nabla)$ is of Ricci type with $M$ simply connected there exists $\left(P, \omega^{P}\right)$ symplectic of dimension 2 higher with a flat connection $\nabla^{P}$ so that $(M, \omega, \nabla)$ is obtained from $\left(P, \omega^{P}, \nabla^{P}\right)$ by reduction.

The manifold $P$ is obtained as the product $P=N \times \mathbb{R}$ of a contact manifold $N$ and the real line $R$. The manifold $N$ is the holonomy bundle over $M$ corresponding to a connection defined on the $S p\left(\mathbb{R}^{2 n+2}, \Omega^{\prime}\right)$-principal bundle

$$
B^{\prime}(M)=B(M) \times_{S p\left(\mathbb{R}^{2 n}, \Omega\right)} S p\left(\mathbb{R}^{2 n+2}, \Omega^{\prime}\right)
$$

with projection $\pi^{\prime}: B(M)^{\prime} \rightarrow M$, where $B(M) \stackrel{\pi}{\rightarrow} M$ is the $S p\left(\mathbb{R}^{2 n}, \Omega\right)$-principal bundle of symplectic frames over $M$ and where we inject the symplectic group $S p\left(\mathbb{R}^{2 n}, \Omega\right)$ into $S p\left(\mathbb{R}^{2 n+2}, \Omega^{\prime}\right)$ as the set of matrices

$$
\tilde{j}(A)=\left(\begin{array}{cc}
I_{2} & 0 \\
0 & A
\end{array}\right) \quad A \in S p\left(\mathbb{R}^{2 n}, \Omega\right) .
$$

The connection 1-form $\alpha^{\prime}$ on $B^{\prime}(M)$ is characterised by the fact that

$$
\alpha_{[\xi, 1]}^{\prime}\left(\left[\bar{X}^{h o r}, 0\right]\right)=\alpha_{\xi}\left(\bar{X}^{h o r}\right) .
$$

where

$$
\alpha_{\xi}\left(\bar{X}^{h o r}\right)=\left(\begin{array}{ccc}
0 & \frac{-\omega_{x}(u, X)}{2(n+1)(2 n+1)} & \frac{-\widetilde{\rho(X)}(\xi)}{2(n+1)} \\
0 & 0 & -\widetilde{X}(\xi) \\
\tilde{X}(\xi) & \frac{-\widetilde{\rho(X)}(\xi)}{2(n+1)} & 0
\end{array}\right)
$$

where $X \in T_{x} M$ with $x=\pi(\xi)$ and $\bar{X}^{\text {hor }}$ is the horizontal lift of $X$ in $\left.T_{\xi} B(M)\right)$.

The equations satisfied by a Ricci-type connection imply that the curvature 2 -form of the connection 1 -form $\alpha^{\prime}$ is equal to $-2 \tilde{A}^{\prime} \pi^{\prime *} \omega$ where $\tilde{A}^{\prime}$ is the unique $S p\left(\mathbb{R}^{2 n+2}, \Omega^{\prime}\right)$-equivariant extension of $\tilde{A}$ to $B^{\prime}(M)$; and this curvature 2 -form is invariant by parallel transport $\left(d^{\alpha^{\prime}} \operatorname{curv}\left(\alpha^{\prime}\right)=0\right)$.

Thus the holonomy algebra of $\alpha^{\prime}$ is of dimension 1 . Assume $M$ is simply connected. The holonomy bundle of $\alpha^{\prime}$ is a circle or a line bundle over $M, N \stackrel{\pi^{\prime}}{\rightarrow} M$. This bundle has a natural contact structure $\nu$ given by the restriction to $N \subset B(M)^{\prime}$ of the 1 -form $-\frac{1}{2} \alpha^{\prime}$ (viewed as real valued since it is valued in a 1-dimensional algebra). One has $d \nu=\pi^{\prime *} \omega$.

The symplectic manifold with connection $\left(P, \omega^{P}, \nabla^{P}\right)$ is then obtained by an induction procedure that we shall expose in a more general setting in Section 7.1.

\section{$5 \quad$ Special symplectic connections}

The striking rigidity results from Section 4 on Ricci-type connections turn out to be a special case of a much more general phenomenon. As we saw, a connection of Ricci-type can be obtained by a symplectic reduction of a symplectic vector space with a flat symplectic connection. This implies, for example, that the local moduli space of such connections is finite dimensional. 
As it turns out, this is merely a special case of a much broader phenomenon. Indeed, there are many more geometric structures which can be characterised in similar terms. For this, we call a symplectic connection on $(M, \omega)$ with $\operatorname{dim} M \geq 4$ special symplectic if it belongs to one of the following classes.

(i) Connections of Ricci-type (cf. Section 4)

\section{(ii) Bochner-Kähler and Bochner-bi-Lagrangian connections}

If the symplectic form is the Kähler form of a (pseudo-)Kähler metric, then its curvature decomposes into the Ricci curvature and the Bochner curvature ([12]). If the latter vanishes, then (the Levi-Civita connection of) this metric is called Bochner-Kähler.

Similarly, if the manifold is equipped with a bi-Lagrangian structure, i.e. two complementary Lagrangian distributions, then the curvature of a symplectic connection for which both distributions are parallel decomposes into the Ricci curvature and the Bochner curvature. Such a connection is called Bochner-bi-Lagrangian if its Bochner curvature vanishes.

For results on Bochner-Kähler and Bochner-bi-Lagrangian connections, see [15] and [30] and the references cited therein.

\section{(iii) Connections with special symplectic holonomy}

A symplectic connection is said to have special symplectic holonomy if its holonomy is contained in a proper absolutely irreducible subgroup of the symplectic group.

The special symplectic holonomies have been classified in [35] and further investigated in [14], [23], [40], [41], [42].

At first, it may seem unmotivated to collect all these structures in one definition, but we shall provide ample justification for doing so. Indeed, there is a beautiful link between special symplectic connections and parabolic contact geometry.

For this, consider a simple Lie group $\mathrm{G}$ with Lie algebra $\mathfrak{g}$. We say that $\mathfrak{g}$ is 2 -gradable, if $\mathfrak{g}$ contains the root space of a long root. This is equivalent to saying that there is a decomposition as a graded vector space

$$
\mathfrak{g}=\mathfrak{g}^{-2} \oplus \mathfrak{g}^{-1} \oplus \mathfrak{g}^{0} \oplus \mathfrak{g}^{1} \oplus \mathfrak{g}^{2}, \quad \text { and } \quad\left[\mathfrak{g}^{i}, \mathfrak{g}^{j}\right] \subset \mathfrak{g}^{i+j},
$$

with $\operatorname{dim} \mathfrak{g}^{ \pm 2}=1$. Indeed, there is a (unique) element $H_{\alpha_{0}} \in\left[\mathfrak{g}^{-2}, \mathfrak{g}^{2}\right] \subset \mathfrak{g}^{0}$ such that $\mathfrak{g}^{i}$ is the eigenspace of $\operatorname{ad}\left(H_{\alpha_{0}}\right)$ with eigenvalue $i=-2, \ldots, 2$, and any non-zero element of $\mathfrak{g}^{ \pm 2}$ is a long root vector.

Denote by $\mathfrak{p}:=\mathfrak{g}^{0} \oplus \mathfrak{g}^{1} \oplus \mathfrak{g}^{2} \leq \mathfrak{g}$ and let $\mathrm{P} \subset \mathrm{G}$ be the corresponding connected Lie subgroup. It follows that the homogeneous space $\mathscr{C}:=\mathrm{G} / \mathrm{P}$ carries a canonical G-invariant contact structure which is determined by the $\mathrm{Ad}_{\mathrm{P}}$-invariant distribution $\mathfrak{g}^{-1} \bmod \mathfrak{p} \subset \mathfrak{g} / \mathfrak{p} \cong T \mathscr{C}$. In fact, we may regard $\mathscr{C}$ as the projectivisation of the adjoint orbit of a maximal root vector. That is, we view $\mathscr{C} \subset \mathbb{P}^{o}(\mathfrak{g})$ where $\mathbb{P}^{o}(V)$ denotes the set of oriented lines through 0 of a vector space $V$, so that $\mathbb{P}^{o}(V)$ is the sphere in $V$.

Each $a \in \mathfrak{g}$ induces an action field $a^{*}$ on $\mathscr{C}$ with flow $\mathrm{T}_{a}:=\exp (\mathbb{R} a) \subset \mathrm{G}$ which hence preserves the contact structure on $\mathscr{C}$. Let $\mathscr{C}_{a} \subset \mathscr{C}$ be the open subset on which $a^{*}$ is transversal to the contact distribution. There is a unique contact form $\alpha \in \Omega^{1}\left(\mathscr{C}_{a}\right)$ determined by the equations that $\alpha\left(a^{*}\right) \equiv 1$. That is, $a^{*}$ is a Reeb vector field of the contact form $\alpha$. 
We can cover $\mathscr{C}_{a}$ by open sets $U$ such that the local quotient $M_{U}:=\mathrm{T}_{a}^{l o c} \backslash U$, i.e. the quotient of $U$ by a sufficiently small neighbourhood of the identity in $\mathrm{T}_{a}$, is a manifold. Then $M_{U}$ inherits a canonical symplectic structure $\omega \in \Omega^{2}\left(M_{U}\right)$ such that $\pi^{*}(\omega)=d \alpha$ for the canonical projection $\pi: U \rightarrow M_{U}$.

It is now our aim to construct a connection on $M_{U}$ which is 'naturally' associated to the given structure. For this, we let $\mathrm{G}_{0} \subset \mathrm{G}$ be the connected subgroup with Lie algebra $\mathfrak{g}^{0} \leq \mathfrak{g}$. Since $\mathfrak{g}^{0} \leq \mathfrak{p}$ and hence $\mathrm{G}_{0} \subset \mathrm{P}$, it follows that we have a fibration

$$
\mathrm{P} / \mathrm{G}_{0} \longrightarrow \mathrm{G} / \mathrm{G}_{0} \longrightarrow \mathscr{C}=\mathrm{G} / \mathrm{P}
$$

In fact, we may interpret $\mathrm{G} / \mathrm{G}_{0}:=\left\{(\alpha, v) \in T_{p}^{*} \mathscr{C} \times T_{p} \mathscr{C} \mid p \in \mathscr{C}, \alpha\left(\mathscr{D}_{p}\right)=0, \alpha(v)=1\right\}$, where $\mathscr{D} \subset T \mathscr{C}$ denotes the contact distribution. Thus, given $a \in \mathfrak{g}$, then for each $p \in \mathscr{C}_{a}$ we may regard the pair $\left(\alpha_{p}, a_{p}^{*}\right)$ from above as a point in $\mathrm{G} / \mathrm{G}_{0}$, i.e., we have a canonical embedding $\imath: \mathscr{C}_{a} \hookrightarrow \mathrm{G} / \mathrm{G}_{0}$.

Let $\Gamma_{a}:=\pi^{-1}\left(\imath\left(\mathscr{C}_{a}\right)\right) \subset \mathrm{G}$ where $\pi: \mathrm{G} \rightarrow \mathrm{G} / \mathrm{G}_{0}$ is the canonical projection. Then the restriction $\pi: \Gamma_{a} \rightarrow \imath\left(\mathscr{C}_{a}\right) \cong \mathscr{C}_{a}$ becomes a principal $\mathrm{G}_{0}$-bundle.

Consider the Maurer-Cartan form $\mu:=g^{-1} d g \in \Omega^{1}(\mathrm{G}) \otimes \mathfrak{g}$ which we decompose according to (24) as $\mu=\sum_{i=-2}^{2} \mu_{i}$ with $\mu_{i} \in \Omega^{1}(\mathrm{G}) \otimes \mathfrak{g}^{i}$. Then we can show the following.

Proposition 5.1 [22] Let $a \in \mathfrak{g}$ be such that $\mathscr{C}_{a} \subset \mathscr{C}$ is non-empty, define the action field $a^{*} \in \mathfrak{X}(\mathscr{C})$ and the principal $\mathrm{G}_{0}$-bundle $\pi: \Gamma_{a} \rightarrow \mathscr{C}_{a}$ with $\Gamma_{a} \subset \mathrm{G}$ from above. Then we have the following.

(i) The restriction of the components $\mu_{0}+\mu_{-1}+\mu_{-2}$ of the Maurer-Cartan form to $\Gamma_{a}$ yields a pointwise linear isomorphism $T \Gamma_{a} \rightarrow \mathfrak{g}^{0} \oplus \mathfrak{g}^{-1} \oplus \mathfrak{g}^{-2}$.

(ii) There is a linear map $R: \mathfrak{g}^{0} \rightarrow \Lambda^{2}\left(\mathfrak{g}^{1}\right)^{*} \otimes \mathfrak{g}^{0}$ and a smooth function $\rho: \Gamma_{a} \rightarrow \mathfrak{g}^{0}$ with the following property. If we define the differential forms $\kappa \in \Omega^{1}\left(\Gamma_{a}\right), \theta \in \Omega^{1}\left(\Gamma_{a}\right) \otimes \mathfrak{g}^{1}$ and $\eta \in \Omega^{1}\left(\Gamma_{a}\right) \otimes \mathfrak{g}^{0}$ by the equation

$$
\mu_{0}+\mu_{-1}+\mu_{-2}=-2 \kappa\left(\frac{1}{2} e_{-2}+\rho\right)+\theta+\eta
$$

for a fixed element $0 \neq e_{-2} \in \mathfrak{g}^{-2}$, then the following equations hold:

$$
d \kappa=\frac{1}{2}<e_{-2},[\theta, \theta]>
$$

and

$$
\begin{aligned}
& d \theta+\eta \wedge \theta=0, \\
& d \eta+\frac{1}{2}[\eta, \eta]=R_{\rho}(\theta \wedge \theta) .
\end{aligned}
$$

Since the Maurer-Cartan form and hence $\kappa, \theta$ and $\eta$ are invariant under the left action of the subgroup $\mathrm{T}_{a} \subset \mathrm{G}$, we immediately get the following

Corollary 5.2 [22] On $\mathrm{T}_{a} \backslash \Gamma_{a}$, there is a coframing $\eta+\theta \in \Omega^{1}\left(\mathrm{~T}_{a} \backslash \Gamma_{a}\right) \otimes\left(\mathfrak{g}^{0} \oplus \mathfrak{g}^{1}\right)$ satisfying the structure equations (27) for a suitable function $\rho: \mathrm{T}_{a} \backslash \Gamma_{a} \rightarrow \mathfrak{g}^{0}$.

Thus, we could, in principle, regard $\theta$ and $\eta$ as the tautological and the connection 1-form, respectively, of a connection on the principal bundle $\mathrm{T}_{a} \backslash \Gamma_{a} \rightarrow \mathrm{T}_{a} \backslash \Gamma_{a} / \mathrm{G}_{0}$ whose curvature is represented by $R_{\rho}$. However, $\mathrm{T}_{a} \backslash \Gamma_{a} / \mathrm{G}_{0} \cong \mathrm{T}_{a} \backslash \mathscr{C}_{a}$ will in general be neither Hausdorff nor locally Euclidean, so the notion of a principal bundle cannot be defined globally. 
The way out of this difficulty is to consider local quotients only, i.e., we restrict to sufficiently small open subsets $U \subset \mathscr{C}_{a}$ for which the local quotient $T_{a}^{l o c} \backslash U$ is a manifold. Clearly, $\mathscr{C}_{a}$ can be covered by such open cells.

Moreover, if we describe explicitly the curvature endomorphisms $R_{\rho}$ for $\rho \in \mathfrak{g}^{0}$, then one can show that - depending on the choice of the 2-gradable simple Lie algebra $\mathfrak{g}$ - the connections constructed above satisfy one of the conditions for a special symplectic connection mentioned before.

More precisely, we have the following

Theorem 5.3 [22] Let $\mathfrak{g}$ be a simple 2-gradable Lie algebra with $\operatorname{dim} \mathfrak{g} \geq 14$, and let $\mathscr{C} \subset \mathbb{P}^{\circ}(\mathfrak{g})$ be the projectivisation of the adjoint orbit of a maximal root vector. Let a $\in \mathfrak{g}$ be such that $\mathscr{C}_{a} \subset \mathscr{C}$ is non-empty, and let $\mathrm{T}_{a}=\exp (\mathbb{R} a) \subset \mathrm{G}$. If for an open subset $U \subset \mathscr{C}_{a}$ the local quotient $M_{U}=\mathrm{T}_{a}^{\text {loc }} \backslash U$ is a manifold, then $M_{U}$ carries a special symplectic connection.

The dimension restriction on $\mathfrak{g}$ guarantees that $\operatorname{dim} M_{U} \geq 4$ and rules out the Lie algebras of type $A_{1}, A_{2}$ and $B_{2}$.

The type of special symplectic connection on $M_{U}$ is determined by the Lie algebra $\mathfrak{g}$. In fact, there is a one-to-one correspondence between the various conditions for special symplectic connections and simple 2-gradable Lie algebras. More specifically, if the Lie algebra $\mathfrak{g}$ is of type $A_{n}$, then the connections in Theorem 5.3 are Bochner-Kähler of signature $(p, q)$ if $\mathfrak{g}=\mathfrak{s u}(p+1, q+1)$ or Bochner-bi-Lagrangian if $\mathfrak{g}=\mathfrak{s l}(n, \mathbb{R})$; if $\mathfrak{g}$ is of type $C_{n}$, then $\mathfrak{g}=\mathfrak{s p}(n, \mathbb{R})$ and these connections are of Ricci-type; if $\mathfrak{g}$ is a 2 -gradable Lie algebra of one of the remaining types, then the holonomy of $M_{U}$ is contained in one of the special symplectic holonomy groups. Also, for two elements $a, a^{\prime} \in \mathfrak{g}$ for which $\mathscr{C}_{a}, \mathscr{C}_{a^{\prime}} \subset \mathscr{C}$ are nonempty, the corresponding connections from Theorem 5.3 are equivalent if and only if $a^{\prime}$ is G-conjugate to a positive multiple of $a$.

If $\mathrm{T}_{a} \cong S^{1}$ then $\mathrm{T}_{a} \backslash \mathscr{C}_{a}$ is an orbifold which carries a special symplectic orbifold connection by Theorem 5.3. Hence it may be viewed as the "standard orbifold model" for (the adjoint orbit of) $a \in \mathfrak{g}$. For example, in the case of positive definite Bochner-Kähler metrics, we have $\mathscr{C} \cong S^{2 n+1}$, and for connections of Ricci-type, we have $\mathscr{C} \cong \mathbb{R} \mathbb{P}^{2 n+1}$. Thus, in both cases the orbifolds $\mathrm{T}_{a} \backslash \mathscr{C}$ are weighted projective spaces if $\mathrm{T}_{a} \cong S^{1}$, hence the standard orbifold models $\mathrm{T}_{a} \backslash \mathscr{C}_{a} \subset \mathrm{T}_{a} \backslash \mathscr{C}$ are open subsets of weighted projective spaces.

Surprisingly, the connections from Theorem 5.3 exhaust all special symplectic connections, at least locally. Namely we have the following

Theorem 5.4 [22] Let $(M, \omega)$ be a symplectic manifold with a special symplectic connection of class $C^{4}$, and let $\mathfrak{g}$ be the Lie algebra associated to the special symplectic condition as above.

(i) Then there is a principal $\hat{\mathrm{T}}$-bundle $\hat{M} \rightarrow M$, where $\hat{\mathrm{T}}$ is a one dimensional Lie group which is not necessarily connected, and this bundle carries a principal connection with curvature $\omega$.

(ii) Let $\mathrm{T} \subset \hat{\mathrm{T}}$ be the identity component. Then there is an $a \in \mathfrak{g}$ such that $\mathrm{T} \cong \mathrm{T}_{a} \subset \mathrm{G}$, and a $\mathrm{T}_{a}$-equivariant local diffeomorphism $\hat{\imath}: \hat{M} \rightarrow \mathscr{C}_{a}$ which for each sufficiently small open subset $V \subset \hat{M}$ induces a connection preserving diffeomorphism $\imath: \mathrm{T}^{l o c} \backslash V \rightarrow \mathrm{T}_{a}^{\text {loc }} \backslash U=M_{U}$, where $U:=\hat{\imath}(V) \subset \mathscr{C}_{a}$ and $M_{U}$ carries the connection from Theorem 5.3.

The situation in Theorem 5.4 can be illustrated by the following commutative diagram, where the vertical maps are quotients by the indicated Lie groups, and $\mathrm{T} \backslash \hat{M} \rightarrow M$ is a regular covering. 


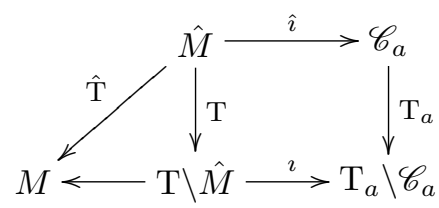

In fact, one might be tempted to summarize Theorems 5.3 and 5.4 by saying that for each $a \in \mathfrak{g}$, the quotient $\mathrm{T}_{a} \backslash \mathscr{C}_{a}$ carries a canonical special symplectic connection, and the map $\imath: \mathrm{T} \backslash \hat{M} \rightarrow \mathrm{T}_{a} \backslash \mathscr{C}_{a}$ is a connection preserving local diffeomorphism. If $\mathrm{T}_{a} \backslash \mathscr{C}_{a}$ is a manifold or an orbifold, then this is indeed correct. In general, however, $\mathrm{T}_{a} \backslash \mathscr{C}_{a}$ may be neither Hausdorff nor locally Euclidean, hence one has to formulate these results more carefully.

As consequences, we obtain the following

Corollary 5.5 All special symplectic connections of $C^{4}$-regularity are analytic, and the local moduli space of these connections is finite dimensional, in the sense that the germ of the connection at one point up to 3 rd order determines the connection entirely. In fact, the generic special symplectic connection associated to the Lie algebra $\mathfrak{g}$ depends on $(\operatorname{rk}(\mathfrak{g})-1)$ parameters.

Moreover, the Lie algebra $\mathfrak{s}$ of affine vector fields, i.e., vector fields on $M$ whose flow preserves the connection, is isomorphic to stab $(a) /(\mathbb{R} a)$ with $a \in \mathfrak{g}$ from Theorem 5.4, where $\operatorname{stab}(a)=\{x \in \mathfrak{g} \mid$ $[x, a]=0\}$. In particular, $\operatorname{dim} \mathfrak{s} \geq \operatorname{rk}(\mathfrak{g})-1$ with equality implying that $\mathfrak{s}$ is abelian.

When counting the parameters in the above corollary, we regard homothetic special symplectic connections as equal, i.e. $(M, \omega, \nabla)$ is considered equivalent to $\left(M, e^{t_{0}} \omega, \nabla\right)$ for all $t_{0} \in \mathbb{R}$.

We can generalize Theorem 5.4 and Corollary 5.5 easily to orbifolds. Indeed, if $M$ is an orbifold with a special symplectic connection, then we can write $M=\hat{T} \backslash \hat{M}$ where $\hat{M}$ is a manifold and $\hat{\mathrm{T}}$ is a one dimensional Lie group acting properly and locally freely on $\hat{M}$, and there is a local diffeomorphism $\hat{\imath}: \hat{M} \rightarrow \mathscr{C}_{a}$ with the properties stated in Theorem 5.4.

There is a remarkable similarity between the cones $\mathscr{C}_{i} \subset \mathfrak{g}_{i}, i=1,2$, for the simple Lie algebras $\mathfrak{g}_{1}:=\mathfrak{s u}(n+1,1)$ and $\mathfrak{g}_{2}:=\mathfrak{s p}(n, \mathbb{R})$. Namely, $\mathscr{C}_{1}=S^{2 n+1}$ with the standard $C R$-structure, and $\mathfrak{g}_{1}$ is the Lie algebra of the group $\mathrm{SU}(n+1,1)$ of $C R$-isomorphisms of $S^{2 n+1}$ [30]. On the other hand, $\mathscr{C}_{2}=\mathbb{R} \mathbb{P}^{2 n+1}$, regarded as the lines in $\mathbb{R}^{2 n+2}$ with the projectivised action of $\mathfrak{s p}(n+1, \mathbb{R})$ on $\mathbb{R}^{2 n+2}$. Thus, $\mathscr{C}_{1}$ is the universal cover of $\mathscr{C}_{2}$, so that the local quotients $\mathrm{T}_{a} \backslash \mathscr{C}_{a}$ are related. In fact, we have the following result.

Proposition 5.6 [38] Consider the action of the 2-gradable Lie algebras $\mathfrak{g}_{1}:=\mathfrak{s u}(n+1,1)$ and $\mathfrak{g}_{2}:=$ $\mathfrak{s p}(n+1, \mathbb{R})$ on the projectivised orbits $\mathscr{C}_{1}$ and $\mathscr{C}_{2}$, respectively. Then the following are equivalent.

(i) For $a_{i} \in \mathfrak{g}_{i}$ the actions of $\mathrm{T}_{a_{i}} \subset \mathrm{G}_{i}$ on $\mathscr{C}_{i}$ are conjugate for $i=1,2$,

(ii) $a_{i} \in \mathfrak{u}(n+1)$ where $\mathfrak{u}(n+1) \subset \mathfrak{g}_{i}$ for $i=1,2$ via the two standard embeddings.

This together with the preceding results yields the following

Theorem 5.7 [38]

(i) Let $(M, \omega, \nabla)$ be a symplectic manifold with a connection of Ricci type, and suppose that the corresponding element $A \in \mathfrak{s} \mathfrak{p}(n+1, \mathbb{R})$ from Theorem 4.6 is conjugate to an element of $\mathfrak{u}(n+1) \subset$ $\mathfrak{s p}(n+1, \mathbb{R})$. Then $M$ carries a canonical Bochner-Kähler metric whose Kähler form is given by $\omega$. 
(ii) Conversely, let $(M, \omega, J)$ be a Bochner-Kähler metric such that the element $a \in \mathfrak{s u}(n+1,1)$ from Theorem 5.4 is conjugate to an element of $\mathfrak{u}(n+1) \subset \mathfrak{s u}(n+1,1)$. Then $(M, \omega)$ carries a canonical connection of Ricci-type.

Note that in [15], Bochner-Kähler metrics have been locally classified. In this terminology, the Bochner-Kähler metrics in the above theorem are called Bochner-Kähler metrics of type I.

\section{Symplectic twistor space and Ricci-type connections}

In this section we present a result of Vaisman [46] which shows how the Ricci-type condition on the curvature of a symplectic connection can be seen as an integrability condition for an associated almost complex structure on the total space of a bundle over the symplectic manifold. It is far from clear what is the geometrical significance of this complex structure. Preliminary studies of its properties have been made in the PhD theses of Albuquerque [1] and Stiénon [43].

\subsection{Compatible almost complex structures}

Let $(V, \Omega)$ be a finite-dimensional real symplectic vector space. We denote by $S p(V, \Omega)$ the real symplectic group of linear transformations $g$ of $V$ which preserve $\Omega$. Any two symplectic vector spaces of the same dimension are isomorphic, and $S p(V, \Omega)$ acts freely and transitively on the set of isomorphisms from $(V, \Omega)$ to any other symplectic vector space of the same dimension by composition on the right.

The Lie algebra $\mathfrak{s p}(V, \Omega)$ of $S p(V, \Omega)$ consists of all linear endomorphisms $\xi$ of $V$ satisfying

$$
\Omega(\xi u, v)+\Omega(u, \xi v)=0, \quad \forall u, v \in V .
$$

This condition is equivalent to saying that $B_{\xi}(u, v)=\Omega(u, \xi v)$ defines a symmetric bilinear form $B_{\xi}$ on $V$. Conversely, any symmetric bilinear form on $V$ defines an element of $\mathfrak{s p}(V, \Omega)$. So as vector spaces $\mathfrak{s p}(V, \Omega)$ and $S^{2} V^{*}$ are isomorphic. In fact, if we consider the natural actions of $S p(V, \Omega)$ on both spaces we have

$$
\left(g \cdot B_{\xi}\right)(u, v)=B_{\xi}\left(g^{-1} u, g^{-1} v\right)=\Omega\left(g^{-1} u, \xi g^{-1} v\right)=\Omega\left(u, g \xi g^{-1} v\right)=B_{A d_{g} \xi}(u, v)
$$

so that the adjoint representation of $S p(V, \Omega)$ is isomorphic to the natural representation of $S p(V, \Omega)$ on $S^{2} V^{*} \cong S^{2} V$.

A compatible almost complex structure $j$ on $(V, \Omega)$ is an element of $\mathfrak{s p}(V, \Omega)$ with $j^{2}=-1$ such that $B_{j}$ is positive definite and we denote the set of compatible almost complex structures by $j(V, \Omega)$. $S p(V, \Omega)$ acts transitively on $j(V, \Omega)$ by conjugation. If we drop the positivity condition, then $S p(V, \Omega)$ has a finite number of orbits distinguished by the signature of $B_{j}$. For our purpose any orbit would do, we choose the positive orbit for convenience.

If $j$ is a compatible almost complex structure on $(V, \Omega)$ then $V$ becomes a complex vector space by defining $(a+i b) v=a v+b j v, \forall a, b \in \mathbb{R}$. Further $\langle u, v\rangle_{j}=B_{j}(u, v)-i \Omega(u, v)$ defines a positive Hermitean structure on $V$ as a complex vector space. Under the action of $S p(V, \Omega)$ above, it is clear that the stabiliser of $j$ is the unitary group $U(V, \Omega, j)$ of this Hermitean structure so that $j(V, \Omega) \cong$ $S p(V, \Omega) / U(V, \Omega, j)$ as homogeneous spaces. 


\subsection{Geometry of $j(V, \Omega)$}

Since $j(V, \Omega)$ is a homogeneous space, the tangent space at $j$ can be identified with the quotient of Lie algebras $\mathfrak{s p}(V, \Omega) / \mathfrak{u}(V, \Omega, j) \cdot \mathfrak{u}(V, \Omega, j)$ consists of all elements of $\mathfrak{s p}(V, \Omega)$ which commute with $j$ so the set of elements which anticommute with $j$ form a complementary subspace

$$
\mathfrak{m}_{j}=\{\xi \in \mathfrak{s p}(V, \Omega) \mid \xi j+j \xi=0\} .
$$

Since $j \xi j \in \mathfrak{s p}(V, \Omega)$ for $\xi \in \mathfrak{s p}(V, \Omega)$ we have a decomposition

$$
\xi=\frac{1}{2}(\xi-j \xi j)+\frac{1}{2}(\xi+j \xi j)
$$

with $\xi-j \xi j \in \mathfrak{u}(V, \Omega, j)$ and $\xi+j \xi j \in \mathfrak{m}_{j}$. This shows that $\mathfrak{s p}(V, \Omega)=\mathfrak{u}(V, \Omega, j)+\mathfrak{m}_{j}$ is a direct sum and so we have a natural isomorphism $T_{j} j(V, \Omega) \cong \mathfrak{m}_{j}$.

The product of two endomorphisms which anticommute with $j$ must commute with $j$ so $\left[\mathfrak{m}_{j}, \mathfrak{m}_{j}\right] \subset$ $\mathfrak{u}(V, \Omega, j)$ and hence $S p(V, \Omega) / U(V, \Omega, j)$ is a symmetric space. In fact this is a realisation of the Siegel domain for the symplectic group. The canonical connection is torsion free and any invariant object is parallel.

If we take $\xi \in \mathfrak{m}_{j}$ then $j \xi=\frac{1}{2}[j, \xi] \in \mathfrak{m}_{j}$ and, as $j$ varies, $\xi \mapsto j \xi$ defines an almost complex structure on $S p(V, \Omega) / U(V, \Omega, j)$ which is clearly $S p(V, \Omega)$-invariant. Being invariant it is parallel and being parallel for a torsion-free connection it is integrable and gives the invariant complex structure of $j(V, \Omega)$ as a Hermitean symmetric space.

If $j$ is an endomorphism of $V$ with $j^{2}=-1$ then $j$ extends complex linearly to the complexification $V^{\mathbb{C}}$ and has $\pm i$ as eigenvalues with eigenspaces $V^{ \pm}$. Then $V^{\mathbb{C}}=V^{+}+V^{-}$is a direct sum and the projections $j^{ \pm}$onto these subspaces are given by $j^{ \pm}=\frac{1}{2}(1 \mp i j)$.

Recall that $\mathfrak{s p}(V, \Omega)$ coincides with $S^{2} V^{*} \cong S^{2} V$ as representations. $j$ acts on both of these as an element of the Lie algebra, and it is clear that the eigenvalues of $j$ on $S^{2} V^{\mathbb{C}}$ will be sums of eigenvalues of $j$ on $V^{\mathbb{C}}$. Thus they are $0, \pm 2 i$ and the same must hold on $\mathfrak{s p}(V, \Omega)^{\mathbb{C}}$. The zero eigenspace corresponds with $\mathfrak{u}(V, \Omega, j)^{\mathbb{C}}$ and the $\pm 2 i$ eigenspaces split $\mathfrak{m}_{j}^{\mathbb{C}}$ into $\mathfrak{m}_{j}^{+}$and $\mathfrak{m}_{j}^{-}$. These are the $\pm i$ eigenspaces of multiplication by $j$ on the left which defined the complex structure. Thus $\mathfrak{m}_{j}^{+}$is the space of $(1,0)$ tangent vectors to $j(V, \Omega)$ at $j$. Since there is no $4 i$ eigenspace on $\mathfrak{s p}(V, \Omega)^{\mathbb{C}}$ we have $\left[\mathfrak{m}_{j}^{+}, \mathfrak{m}_{j}^{+}\right]=0$ and since there is no $3 i$ eigenspace on $V^{\mathbb{C}}, \mathfrak{m}_{j}^{+} V^{+}=0$. Note that $\mathfrak{m}_{j}^{-} V^{+}=V^{-}$so the condition $\mathfrak{m}_{j}^{+} V^{+}=0$ is a compatibility condition between the complex structures on $V$ and $j(V, \Omega)$ which distinguishes the two possible invariant complex structures on $j(V, \Omega)$ and allows us to choose one in preference to the other.

\subsection{The bundle $J(M, \omega)$ of almost complex structures}

We fix a symplectic vector space $(V, \Omega)$ and consider a symplectic manifold $(M, \omega)$ of the same dimension as $V$. A symplectic frame at $x \in M$ is a symplectic isomorphism $p$ from $(V, \Omega)$ to $\left(T_{x} M, \omega_{x}\right)$. We denote the set of symplectic frames at $x$ by $S p(M, \omega)_{x}$ and the disjoint union of these over $x \in M$ by $S p(M, \omega)$. With the obvious projection map $\pi: S p(M, \omega) \rightarrow M, S p(M, \omega)$ is the symplectic frame bundle of $(M, \omega)$. It is a principal $S p(V, \Omega)$ bundle with $S p(V, \Omega)$ acting on the right by composition.

Denote by $J(M, \omega)$ the bundle on $M$ whose fibre at $x$ is $j\left(T_{x} M, \omega_{x}\right)$. Smooth sections of $J(M, \omega)$ are almost complex structures on $M$ compatible with the symplectic structure so we call $J(M, \omega)$ the bundle of compatible almost complex structures. $J(M, \omega)$ can also be viewed as the associated bundle 
$S p(M, \omega) \times_{S p(V, \Omega)} j(V, \Omega)$ with fibre $j(V, \Omega)$. Since the latter is homogeneous, $J(M, \omega)$ can be identified with the quotient space $S p(M, \omega) / U(V, \Omega, j)$ with the identification given by

$$
S p(M, \omega) / U(V, \Omega, j) \ni p . U(V, \Omega, j) \longleftrightarrow p \circ j \circ p^{-1} \in J(M, \omega)
$$

$\operatorname{Sp}(M, \omega)$ has a $V$-valued 1-form $\theta$ called the soldering form defined by

$$
\theta_{p}(X)=p^{-1}\left(\pi_{*} X\right)
$$

and a vertical bundle $\mathscr{V}$ with

$$
\mathscr{V}_{p}=\operatorname{Ker} \pi_{*}: T_{p} S p(M, \omega) \rightarrow T_{\pi(p)} M
$$

so $\mathscr{V}$ is the kernel of $\theta$. A 1 -form on $S p(M, \omega)$ is called horizontal if it vanishes on $\mathscr{V}$. The components of $\theta$ span the horizontal forms pointwise.

If $\xi \in \mathfrak{s p}(V, \Omega)$ then $\widetilde{\xi}$ is the vertical vectorfield on $\operatorname{Sp}(M, \omega)$ defined by:

$$
\widetilde{\xi}_{p}=\left.\frac{d}{d t} p \circ \exp t \xi\right|_{t=0}
$$

$(p, \xi) \mapsto \widetilde{\xi}_{p}$ identifies the trivial bundle $S p(M, \omega) \times \mathfrak{s p}(V, \Omega)$ with $\mathscr{V}$. Note that the pull-back bundle $\pi^{-1} T M$ to $\operatorname{Sp}(M, \omega)$ is also trivial via the map $(p, X) \mapsto\left(p, p^{-1} X\right)$ so $\theta$ gives a map $T S p(M, \omega) \rightarrow$ $S p(M, \omega) \times V$ and an exact sequence of bundles on $S p(M, \omega)$

$$
0 \longrightarrow S p(M, \omega) \times \mathfrak{s p}(V, \Omega) \longrightarrow T S p(M, \omega) \longrightarrow S p(M, \omega) \times V \longrightarrow 0
$$

Splitting this exact sequence trivialises the tangent bundle of $\operatorname{Sp}(M, \omega)$ and makes calculations with forms particularly easy.

\subsection{Almost symplectic connections}

A principal connection in $\operatorname{Sp}(M, \omega)$ is an almost symplectic connection $\nabla$ in $T M$ (a symplectic connection additionally has vanishing torsion). It is given by an $\mathfrak{s p}(V, \Omega)$-valued 1-form $\alpha$ on $S p(M, \omega)$ which satisfies $\alpha(\widetilde{\xi})=\xi$ and $g^{*} \alpha=\operatorname{Ad}_{g} \alpha$ for all $\xi \in \mathfrak{s p}(V, \Omega)$ and $g \in S p(V, \Omega)$. It follows that $\mathscr{H}=\operatorname{Ker} \alpha$ is a complementary subbundle to $\mathscr{V}$ which we call the horizontal bundle determined by $\alpha$. $\nabla$ and $\alpha$ are related as follows: if $U$ is an open set in $M$ on which there is a local section $s$ of $S p(M, \omega)$ then $s v$ is a vectorfield on $U$ for any fixed $v$ in $V$ and $s^{*} \alpha$ is an $\mathfrak{s p}(V, \Omega)$-valued 1-form on $U$ which are related by

$$
\nabla_{X}(s v)=s\left(\left(s^{*} \alpha\right)(X) v\right)
$$

or, more compactly,

$$
s^{-1} \nabla s=s^{*} \alpha
$$

with the appropriate interpretation of the two sides of the equation.

The components of the forms $\alpha, \theta$ are linearly independent and span the cotangent spaces of $S p(M, \omega)$ at each point so that $S p(M, \omega)$ is parallelisable. This makes the differential geometry on $S p(M, \omega)$ especially simple and is the reason why we shall do calculations on $S p(M, \omega)$ in preference to $M$ or $J(M, \omega)$. 
The torsion and curvature of $\nabla$ lift to $S p(M, \omega)$ as horizontal $V$ - and $\mathfrak{s p}(V, \Omega)$-valued 2-forms $\tau^{\nabla}$ and $\rho^{\nabla}$ given by

$$
\tau_{p}^{\nabla}(X, Y)=p^{-1}\left(T_{\pi(p)}^{\nabla}\left(\pi_{*} X, \pi_{*} Y\right)\right), \quad \rho_{p}^{\nabla}(X, Y)=p^{-1} \circ R_{\pi_{(p)}}^{\nabla}\left(\pi_{*} X, \pi_{*} Y\right) \circ p .
$$

These forms can be computed from the corresponding principal connection $\alpha$ by

$$
\tau^{\nabla}=d \theta+\alpha \wedge \theta, \quad \rho^{\nabla}=d \alpha+\frac{1}{2}[\alpha \wedge \alpha]
$$

where $[\alpha \wedge \alpha]$ denotes simultaneous wedge as forms and Lie bracket of the values.

\subsection{Differential geometry on $J(M, \omega)$}

Let $\pi$ be the projection $J(M, \omega) \rightarrow M$, then $\pi$ is a submersion so $d \pi$ is surjective and we have an exact sequence of vector bundles on $J(M, \omega)$

$$
0 \longrightarrow \mathscr{V} \longrightarrow T J(M, \omega) \stackrel{d \pi}{\longrightarrow} \pi^{-1} T M \longrightarrow 0
$$

where $\mathscr{V}$ now denotes the vertical bundle on $J(M, \omega)$. It follows that if $j \in J(M, \omega)$ then $\mathscr{V}_{j}$ consists of all elements $\xi$ of $\mathfrak{s p}\left(T_{x} M, \omega_{x}\right)$ which anticommute with $j$ where $x=\pi(j)$. We identify $\mathscr{V}_{j}$ with this subspace via the map

$$
\mathfrak{s p}\left(T_{x} M, \omega_{x}\right) \ni \xi \longleftrightarrow \frac{d}{d t} \exp t \xi \circ j \circ \exp -\left.t \xi\right|_{t=0} .
$$

$\operatorname{End}\left(\pi^{-1} T M\right)$ has a tautological section which we denote by $\mathscr{J}$ whose value at $j$ is $j \in \operatorname{End}\left(T_{\pi(j)} M\right)$. If we denote by $\mathfrak{s p}(M, \omega)$ the bundle of Lie algebras whose fibre at $x$ is $\mathfrak{s p}\left(T_{x} M, \omega_{x}\right)$ then $\mathscr{J}$ is a section of $\pi^{-1} \mathfrak{s p}(M, \omega)$. The kernel of ad $\mathscr{J}$ is a bundle $\mathfrak{u}(M, \omega, \mathscr{J})$ of unitary Lie algebras on $J(M, \omega)$. The range of $\operatorname{ad} \mathscr{J}$ is the elements of $\pi^{-1} \mathfrak{s p}(M, \omega)$ which anticommute with $\mathscr{J}$ and so coincides with the tangent space to the fibre, namely the vertical bundle $\mathscr{V}$. Thus

$$
\pi^{-1} \mathfrak{s p}(M, \omega)=\mathfrak{u}(M, \omega, \mathscr{J})+\mathscr{V}
$$

If $\nabla$ is the almost symplectic connection on $T M$ corresponding to a principal connection $\alpha$ then the horizontal distribution is $U(V, \Omega, \mathscr{J})$-invariant and hence projects to a horizontal distribution $\mathscr{H}^{\nabla}$ on $J(M, \omega)$. Our next objective is to identify $\mathscr{H}^{\nabla}$ directly without going via the frame bundle.

The $\mathfrak{s p}(V, \Omega)$ connection form $\alpha$ in $S p(M, \omega)$ pulls back to $\pi^{*} \alpha$ in $\pi^{-1} S p(M, \omega)$ and then pulls back to the reduction as $\alpha$. It has values in $\mathfrak{s p}(V, \Omega)$ rather than the Lie algebra $\mathfrak{u}\left(V, \Omega, j_{0}\right)$ so it is a Cartan connection from the point of view of this reduction. We can split $\alpha$ relative to the decomposition

$$
\mathfrak{s p}(V, \Omega)=\mathfrak{u}\left(V, \Omega, j_{0}\right) \oplus \mathfrak{m}_{j_{0}}
$$

as

$$
\alpha=\alpha^{u}+\alpha^{m}
$$

Obviously, $\alpha^{u}$ is a principal connection in the bundle $S p(M, \omega) \longrightarrow J(M, \omega)$. $\alpha^{m}$ vanishes on vertical vectors for this bundle so is the lift of a $S p(M, \Omega) \times_{U\left(V, \Omega, j_{0}\right)} \mathfrak{m}_{j_{0}}$-valued 1-form. Since this associated bundle is $\mathscr{V}$ we clearly have a $\mathscr{V}$-valued 1 -form on $J(M, \omega)$.

Let $\mathscr{P}$ be the bundle map $T J(M, \omega) \longrightarrow \pi^{-1} \mathfrak{s p}(M, \omega)$ with image $\mathscr{V}$ and kernel $\mathscr{H} \nabla$. Then $\mathscr{P}$ anticommutes with $\mathscr{J}$. Note that $\mathscr{P}$ can be viewed as a $\pi^{-1} \mathfrak{s p}(M, \omega)$-valued 1-form. 
Proposition 6.1 The horizontal lift of $\mathscr{P}$ to $S p(M, \omega)$ is $\alpha^{m}$.

The almost symplectic connection $\nabla$ induces a connection in $\mathfrak{s p}(M, \omega)$ and hence its pull-back $\pi^{-1} \nabla$ induces one in $\pi^{-1} \mathfrak{s p}(M, \omega)$. We can thus take the covariant derivative $\pi^{-1} \nabla \mathscr{J}$ which is also a $\pi^{-1} \mathfrak{s p}(M, \omega)$-valued 1-form. Moreover $\mathscr{J}^{2}=-1$ so $\pi^{-1} \nabla \mathscr{J} \mathscr{J}+\mathscr{J} \pi^{-1} \nabla \mathscr{J}=0$ and hence $\pi^{-1} \nabla \mathscr{J}$ is $\mathscr{V}$-valued. These two 1 -forms are related by

\section{Proposition 6.2}

$$
\pi^{-1} \nabla \mathscr{J}=[\mathscr{P}, \mathscr{J}]
$$

Proof $\mathscr{J}$ lifts to $\pi^{-1} S p(M, \omega)$ as $\widehat{\mathscr{J}}(j, p)=p \circ j \circ p^{-1}$ and so on the reduction is given by $\widehat{\mathscr{J}}(\sigma(p))=$ $j_{0}$. So it is constant. On the reduction the covariant derivative is then $\sigma^{*}\left(d \widehat{\mathscr{J}}+\left[\widehat{\pi}^{*} \alpha, \widehat{\mathscr{J}}\right]\right)=\left[\alpha, j_{0}\right]=$ $\left[\alpha^{m}, j_{0}\right]$.

Finally we can describe the horizontal distribution on $J(M, \omega)$ directly.

\section{Corollary 6.3}

$$
\mathscr{H}^{\nabla}=\left\{X \in T J(M, \omega) \mid \pi^{-1} \nabla_{X} \mathscr{J}=0\right\}
$$

\subsection{The almost complex structures on $J(M, \omega)$}

If $(M, \omega)$ is a symplectic manifold the fibres of $J(M, \omega)$ are diffeomorphic to $j(V, \Omega)$ which has an invariant complex structure, which then transfers to each fibre. The tangent bundle to the fibres $\mathscr{V}$ thus has an endomorphism which gives the almost complex structure of each fibre. Under the identification of $\mathscr{V}$ with endomorphisms of $\pi^{-1} T M$ this agrees with left multiplication by $\mathscr{J}$.

If $\nabla$ is an almost symplectic connection on $(M, \omega)$ then the horizontal distribution $\mathscr{H} \nabla$ is isomorphic via $d \pi$ with $\pi^{-1} T M$ and the latter has an endomorphism $\mathscr{J}$. Since $T J(M, \omega)=\mathscr{V} \oplus \mathscr{H}^{\nabla}$ there is a unique endomorphism $J^{\nabla}$ of $T J(M, \omega)$ which coincides with multiplication by $\mathscr{J}$ on $\mathscr{V}$, and which satisfies $d \pi \circ J^{\nabla}=\mathscr{J} \circ d \pi$. Clearly $J^{\nabla}$ is an almost complex structure on $J(M, \omega)$. Thus we have shown

Proposition 6.4 If $\nabla$ is an almost symplectic connection on the symplectic manifold $(M, \omega)$ then there is a unique almost complex structure $J^{\nabla}$ on $J(M, \omega)$ which satisfies

- $\mathscr{P} \circ J^{\nabla}=\mathscr{J} \circ \mathscr{P}$, or equivalently, $\pi^{-1} \nabla_{J^{\nabla}} \mathscr{J}=\mathscr{J} \pi^{-1} \nabla_{X} \mathscr{J}$ for all $X$;

- $d \pi \circ J^{\nabla}=\mathscr{J} \circ d \pi$.

It is our goal to prove the following theorem.

Theorem 6.5 Let $\nabla$ be a connection on the symplectic manifold $(M, \omega)$.

(i) If $\nabla$ is almost symplectic and $J^{\nabla}$ is integrable then there is a symplectic connection $\nabla^{\prime}$ with $J^{\nabla^{\prime}}=J^{\nabla}$.

(ii) If $\nabla, \nabla^{\prime}$ are symplectic connections then $J^{\nabla^{\prime}}=J^{\nabla}$ implies $\nabla=\nabla^{\prime}$.

(iii) $J^{\nabla}$ is integrable if and only if $\nabla$ is of Ricci-type. 
The first step is to convert the integrability question into a condition on the frame bundle. This is possible since integrability of an almost complex structure is equivalent to its $(1,0)$ forms generating a $d$-closed ideal. Since the map $S p(M, \omega) \rightarrow J(M, \omega)$ is a submersion, the pull-back of forms is an injective map, so any relation amongst forms on $J(M, \omega)$ will hold if and only if it holds amongst their pull-backs to $S p(M, \omega)$. Thus we will have integrability if and only if a pointwise basis for the pull-backs of $(1,0)$ forms generates a $d$-closed ideal on $S p(M, \omega)$.

Lemma 6.6 The pull-backs to $\operatorname{Sp}(M, \omega)$ of $(1,0)$ forms for $J^{\nabla}$ are spanned pointwise by $\alpha^{+}=j^{+} \alpha j^{-}$ and $\theta^{+}=j^{+} \theta$ if $\alpha$ is the connection form for $\nabla$.

See [36] for a proof. An immediate consequence is

Lemma 6.7 $J^{\nabla}$ is integrable if and only if $j^{+} \tau^{\nabla}$ and $j^{+} \rho^{\nabla} j^{-}$are in the ideal generated by $\alpha^{+}, \theta^{+}$.

Proof

$$
j^{+} \tau^{\nabla}-d \theta^{+}=j^{+} \alpha \wedge \theta=j^{+}\left(\alpha\left(j^{+}+j^{-}\right) \wedge \theta\right)=j^{+} \alpha \wedge \theta^{+}+\alpha^{+} \wedge \theta
$$

and

$$
j^{+} \rho^{\nabla} j^{-}-d \alpha^{+}=j^{+} \rho^{\nabla}-d \alpha j^{-}=\frac{1}{2} j^{+}[\alpha, \alpha] j^{-}=\left[\alpha^{u}, \alpha^{+}\right] .
$$

This gives the Lemma immediately.

Proof of Theorem 6.5 (i): If $\nabla$ is almost symplectic with torsion $T^{\nabla}$ then it is easily verified that the connection $\nabla^{\prime}$ defined by

$$
\omega\left(\nabla^{\prime}{ }_{X} Y, Z\right)=\omega\left(\nabla_{X} Y, Z\right)-\frac{1}{2} \omega\left(T^{\nabla}(X, Y), Z\right)+\frac{1}{6} \omega\left(X, T^{\nabla}(Y, Z)\right)+\frac{1}{6} \omega\left(Y, T^{\nabla}(X, Z)\right)
$$

is a symplectic connection. The connection forms $\alpha$ and $\alpha^{\prime}$ on $S p(M, \omega)$ are related by

$$
\Omega\left(\alpha^{\prime} u, v\right)=\Omega(\alpha u, v)-\frac{1}{2} \Omega\left(\tau^{\nabla}(\theta, u), v\right)+\frac{1}{6} \Omega\left(\theta, \tau^{\nabla}(u, v)\right)+\frac{1}{6} \Omega\left(u, \tau^{\nabla}(\theta, v)\right) .
$$

Hence

$$
\begin{aligned}
\Omega\left(\alpha^{\prime+} u, v\right)= & \Omega\left(\alpha^{\prime} j^{-} u, j^{-} v\right) \\
= & \Omega\left(\alpha^{+} u, v\right)-\frac{1}{2} \Omega\left(j^{+} \tau^{\nabla}\left(\theta, j^{-} u\right), v\right) \\
& \quad+\frac{1}{6} \Omega\left(\theta, j^{+} \tau^{\nabla}\left(j^{-} u, j^{-} v\right)\right)+\frac{1}{6} \Omega\left(u, j^{+} \tau^{\nabla}\left(\theta, j^{-} v\right)\right) .
\end{aligned}
$$

Integrability implies that $j^{+} \tau^{\nabla}\left(j^{-} u, j^{-} v\right)=0$ from which one sees that $j^{+} \tau^{\nabla}\left(\theta, j^{-} v\right)=j^{+} \tau^{\nabla}\left(\theta^{+}, j^{-} v\right)$ and hence that $\alpha^{\prime+}$ is a combination of $\alpha^{+}$and $\theta^{+}$so the $(1,0)$ forms of $J^{\nabla}$ and $J^{\nabla^{\prime}}$ agree and hence the almost complex structures are the same.

Proof of Theorem 6.5 (ii): Suppose $\nabla$ and $\nabla^{\prime}$ are both symplectic connections defining the same almost complex structures $J^{\nabla}=J^{\nabla^{\prime}}$, which will be the case if and only if the components of $\alpha^{\prime+}$ are combinations of the components of $\alpha^{+}$and $\theta^{+}$. If $\nabla^{\prime}=\nabla+B$ and we lift $B$ to $S p(M, \omega)$ as

$$
\beta_{p}(X)=p^{-1} \circ B_{\pi(p)}\left(\pi_{*} X\right) \circ p
$$

then $\alpha^{\prime}=\alpha+\beta$ so $J^{\nabla^{\prime}}=J^{\nabla}$ if and only if $\beta^{+}$is a combination of the components of $\alpha^{+}$and $\theta^{+}$, and since $\beta$ is horizontal, this means $\beta^{+}$vanishes on $(0,1)$ vectors. Hence $j^{+} B_{x}\left(j^{-} X\right) j^{-} Y=0$ for every $j \in j\left(T_{x} M, \omega_{x}\right)$. 
Solving this condition can be converted to a problem in representation theory by observing first that it can be re-expressed in terms of the completely symmetric 3-tensor $\underline{B}(X, Y, Z)=\omega(B(X) Y, Z)$ as $\underline{B}\left(j^{-} X, j^{-} Y, j^{-} Z\right)=0$. Since the set of such $j$ is $S p(V, \Omega)$-invariant, this says that $\underline{B}$ takes its values in an invariant subspace in which there is no $3 i$ eigenvalue for any $j$ as an element of the Lie algebra. But $S^{3} V$ is irreducible and the $3 i$ eigenvalue does occur (on $S^{3} V^{+}$) hence the only invariant subspace with no $3 i$ eigenvalue is $\{0\}$. Thus $B=0$ and hence $\nabla^{\prime}=\nabla$.

Proof of Theorem 6.5 (iii): We again write the integrability condition in terms of representation theory as in the proof of injectivity. The point is that $j^{+} \rho^{\nabla} j^{-}$consists of horizontal forms so is in the ideal generated by $(1,0)$ forms if and only if $j^{+} R_{x}^{\nabla}\left(j^{-} X, j^{-} Y\right) j^{-} Z=0$ for all $j \in j\left(T_{x} M, \omega_{x}\right)$. This is again an invariant condition, so it means $R^{\nabla}$ has to take its values in the largest invariant subspace of the space of curvature tensors where there is no $4 i$ eigenvalue of $j$ as an element of the Lie algebra. We know that there are two irreducible components to curvature, and one is the Ricci component which is isomorphic to symmetric 2 -tensors, so only has eigenvalues 0 and $\pm 2 i$. The other irreducible component does have $4 i$ eigenvalues and so for integrability the curvature must be Ricci-type.

\subsection{Generalisations}

The fibres of $J(M, \omega)$ are large which is the reason the integrability condition kills off so much of the curvature. We can try to find subbundles with smaller fibres which are complex submanifolds of the fibres of $J(M, \omega)$ and then play the same game with this smaller bundle. For example we might have a $G$-structure $P \rightarrow M$ for some subgroup $G$ of $S p(V, \Omega)$ and a complex orbit $G / H$ of $G$ on $j(V, \Omega)$. Then $P \times{ }_{G} G / H=P / H$ will have an almost complex structure for each choice of principal $G$-connection and a similar calculation can be performed to determine when it is integrable. If $G$ is small then the curvature and torsion may break into several irreducible pieces more of which may survive the integrability condition. On the other hand as $G$ gets smaller it is harder to have such a $G$-structure

Alternatively, for a given symplectic connection $\nabla$ we can look at the zero-set of the Nijenhuis tensor of $J^{\nabla}$ on $J(M, \omega)$ for a given symplectic connection $\nabla$. If a component of this set turns out to be a complex manifold then it can be seen as a twistorial space over $M$.

Both these approaches lead to interesting examples of twistor spaces in the Riemannian case. Little is yet known in the symplectic case.

\subsection{The bundle $J(M, \omega)$ for spaces of Ricci-type}

In view of Theorem 6.5 it is interesting to describe the twistor space $J(M, \omega)$ for manifolds of Riccitype in more detail. In particular, since manifolds of Ricci-type can always be obtained locally by the reduction process explained in Theorem 4.6, it seems reasonable to expect the bundle $J(M, \omega)$ and its complex structure to arise as a reduction of some sort as well. It is the aim of this section to look at this question.

First of all, consider the twistor space $J\left(\mathbb{R}^{2 n+2}, \Omega^{\prime}\right)$ of $\mathbb{R}^{2 n+2}$ with the canonical symplectic structure $\Omega^{\prime}$, let $A \in \mathfrak{s p}\left(\mathbb{R}^{2 n+2}, \Omega^{\prime}\right)$ and $\Sigma_{A}:=\left\{x \in \mathbb{R}^{2 n+2} \mid \Omega^{\prime}(x, A x)=1\right\}$. We decompose the tangent spaces at $x \in \Sigma_{A} \subset \mathbb{R}^{n+2}$ as

$$
T_{x} \Sigma_{A}=\operatorname{span}(A x) \oplus \mathscr{D}_{x} \quad \text { and } \quad T_{x} \mathbb{R}^{2 n+2}=\operatorname{span}(x, A x) \oplus \mathscr{D}_{x},
$$


where $\mathscr{D}_{x}$ is determined by $\Omega^{\prime}\left(\operatorname{span}(x, A x), \mathscr{D}_{x}\right)=0$. Moreover, we have the canonical projection $\pi: \Sigma_{A} \supset U \rightarrow M^{\text {red }}$ where $U$ is a sufficiently small open subset such that $M^{r e d}=U /(\exp \mathbb{R} A)^{l o c}$ is a manifold, as explained in Section 4.2. Recall that the symplectic structure $\omega$ on $M^{\text {red }}$ is determined by the requirement that $\left.d \pi\right|_{\mathscr{D}_{x}}:\left(\mathscr{D}_{x}, \Omega^{\prime}\right) \rightarrow\left(T_{\pi(x)} M^{\text {red }}, \omega_{\pi(x)}\right)$ becomes a symplectic isomorphism. Moreover, the Ricci-type connection $\nabla$ on $\left(M^{\text {red }}, \omega\right)$ is defined by

$$
\overline{\nabla_{X} Y}=\left(\nabla_{\bar{X}}^{0} \bar{Y}\right)_{\mathscr{D}} \text {, for all vector fields } X, Y \text { on } M^{\text {red }},
$$

where the bar denotes the horizontal lift w.r.t. the distribution $\mathscr{D}$, the subscript $\mathscr{D}$ denotes the image under the canonical projection $T_{x} \mathbb{R}^{2 n+2}=\operatorname{span}(x, A x) \oplus \mathscr{D}_{x} \rightarrow \mathscr{D}_{x}$, and where $\nabla^{0}$ denotes the canonical connection on $\mathbb{R}^{2 n+2}$.

Lemma 6.8 Let $p: J\left(M^{\text {red }}, \omega\right) \rightarrow M^{\text {red }}$ be the twistor fibration. Then the pull-back of $p$ under the map $\pi: \Sigma_{A} \rightarrow M^{\text {red }}$ is given by the fibration $\hat{p}: \hat{Z}_{A} \rightarrow \Sigma_{A}$ where

$$
\hat{Z}_{A}:=\left\{(x, J) \in J\left(\mathbb{R}^{2 n+2}, \Omega^{\prime}\right) \mid x \in \Sigma_{A}, J x=A x, \quad J A x=-x\right\}, \quad \hat{p}(x, J)=x
$$

and the map $\hat{\pi}: \hat{Z}_{A} \rightarrow J\left(M^{\text {red }}, \omega\right)$ is given by

$$
(\hat{\pi}(J))(v):=d \pi(J \bar{v}), \quad \forall v \in T_{\pi(x)} M^{r e d} .
$$

Moreover, $J\left(M^{\text {red }}, \omega\right)$ is the quotient of $\hat{Z}_{A}$ under the (local) action of $\exp (\mathbb{R} A)$ given by

$$
\exp (t A) \cdot(x, J):=(\exp (t A) x, \exp (t A) J \exp (-t A)) .
$$

Proof Let $x \in \Sigma_{A}$ and consider an element $J \in j\left(T_{\pi(x)} M^{\text {red }}, \omega\right)$. Using the symplectic isomorphism $d \pi_{x}:\left(\mathscr{D}_{x}, \Omega^{\prime}\right) \rightarrow\left(T_{\pi(x)} M^{r e d}, \omega\right)$, we see that there is a unique way to extend from $\mathscr{D}_{x}$ to $\mathbb{R}^{2 n+2}$ to give an element $\hat{J} \in j\left(\mathbb{R}^{2 n+2}, \Omega^{\prime}\right)$ with $(x, \hat{J}) \in \hat{Z}_{A}$ and $\hat{\pi}(x, \hat{J})=J$. This shows the first assertion. The second assertion is straightforward.

After having described $J\left(M^{\text {red }}, \omega\right)$ as a manifold as the reduction of $\hat{Z}_{A}$ under the (local) action of $\exp (\mathbb{R} A)$ it would have been nice to see that the twistor almost complex structure on $J\left(M^{r e d}, \omega\right)$ coming from the reduced connection arose as a quotient from the twistor complex structure on $J\left(\mathbb{R}^{2 n+2}, \Omega^{\prime}\right)$ coming from $\nabla^{0}$. Unfortunately this is not the case. The inverse image by $d \hat{\pi}$ of the twistor almost complex structure on $J\left(M^{\text {red }}, \omega\right)$ gives an almost complex structure on the quotient of $T \hat{Z}_{A}$ by $\mathbb{R} \hat{H}_{A}$ and this would be induced from $J^{\nabla^{0}}$ on $J\left(\mathbb{R}^{2 n+2}, \Omega^{\prime}\right)$ only if $J^{\nabla^{0}} T \hat{Z}_{A} \subset T \hat{Z}_{A}+\mathbb{R} J^{\nabla^{0}} \hat{H}_{A}$. A straightforward calculation shows that this last condition is equivalent to

$$
[A, J] \mathscr{D}_{x}=0, \quad \forall(x, J) \in \hat{Z}_{A}
$$

and that this condition is too strong for general $A$.

\section{$7 \quad$ Ricci-flat connections}

\subsection{A construction by induction}

Definition 7.1 A contact quadruple $(M, N, \alpha, \pi)$ is a $2 n$ dimensional smooth manifold $M$, a $2 n+1$ dimensional smooth manifold $N$, a co-oriented contact structure $\alpha$ on $N$ (i.e. $\alpha$ is a 1-form on $N$ such that $\alpha \wedge(d \alpha)^{n}$ is nowhere vanishing), and a smooth submersion $\pi: N \rightarrow M$ with $d \alpha=\pi^{*} \omega$ where $\omega$ is a symplectic 2-form on $M$. 
Definition 7.2 Given a contact quadruple $(M, N, \alpha, \pi)$ the induced symplectic manifold is the $2 n+2$ dimensional manifold

$$
P:=N \times \mathbb{R}
$$

endowed with the (exact) symplectic structure

$$
\mu:=2 e^{2 s} d s \wedge p_{1}^{*} \alpha+e^{2 s} d p_{1}^{*} \alpha=d\left(e^{2 s} p_{1}^{*} \alpha\right)
$$

where $s$ denotes the variable along $\mathbb{R}$ and $p_{1}: P \rightarrow N$ the projection on the first factor.

Induction in the sense of building a $(2 n+2)$-dimensional symplectic manifold from a symplectic manifold of dimension $2 n$ is also considered by Kostant in [32].

\section{Remark 7.3}

- The vector field $S:=\partial_{s}$ on $P$ is such that $i(S) \mu=2 \mathrm{e}^{2 s}\left(p_{1}^{*} \alpha\right)$; hence $L_{S} \mu=2 \mu$ and $S$ is a conformal vector field.

- The Reeb vector field $Z$ on $N$ (i.e. the vector field $Z$ on $N$ such that $i(Z) d \alpha=0$ and $i(Z) \alpha=1)$ lifts to a vector field $E$ on $P$ such that: $p_{1 *} E=Z$ and $d s(E)=0$. Since $i(E) \mu=-d\left(\mathrm{e}^{2 s}\right), E$ is a Hamiltonian vector field on $(P, \mu)$. Furthermore

$$
\begin{aligned}
{[E, S] } & =0 \\
\mu(E, S) & =-2 \mathrm{e}^{2 s} .
\end{aligned}
$$

- Observe also that if $\Sigma=\{y \in P \mid s(y)=0\}$, the reduction of $(P, \mu)$ relative to the constraint manifold $\Sigma$ (which is isomorphic to $N$ ) is precisely $(M, \omega)$.

- For $y \in P$ define $H_{y}\left(\subset T_{y} P\right)=>E, S<^{\perp_{\mu}}$. Then $H_{y}$ is symplectic and $\left(\pi \circ p_{1}\right)_{* y}$ defines a linear isomorphism between $H_{y}$ and $T_{\pi p_{1}(y)} M$. Vector fields on $M$ thus admit "horizontal" lifts to $P$.

We shall now prove that any symplectic connection $\nabla$ on $(M, \omega)$ can be lifted to a symplectic connection on $\left(P, \omega^{P}\right)$ which is Ricci-flat. We shall initially define a connection $\nabla^{P}$ on $P$ induced by $\nabla$.

First some notation:

$p$ denotes the projection $p=\pi \circ p_{1}: P \rightarrow M$.

If $X$ is a vector field on $M, \overline{\bar{X}}$ is the vector field on $P$ such that

$$
\text { (i) } p_{*} \overline{\bar{X}}=X \quad(i i) \quad\left(p_{1}^{*} \alpha\right)(\overline{\bar{X}})=0 \quad(i i i) \quad d s(\overline{\bar{X}})=0 \text {. }
$$

Recall that $E$ is the vector field on $P$ such that

$$
\text { (i) } p_{1 *} E=Z \quad(i i) d s(E)=0 \text {. }
$$

Clearly the values at any point of $P$ of the vector fields $\overline{\bar{X}}, E, S=\partial_{s}$ span the tangent space to $P$ at that point and we have

$$
\left[E, \partial_{s}\right]=0 \quad[E, \overline{\bar{X}}]=0 \quad\left[\partial_{s}, \overline{\bar{X}}\right]=0 \quad[\overline{\bar{X}}, \overline{\bar{Y}}]=\overline{\overline{[X, Y]}}-p^{*} \omega(X, Y) E
$$


The formulas for $\nabla^{P}$ are:

$$
\begin{aligned}
\nabla_{\overline{\bar{X}}}^{P} \overline{\bar{Y}} & =\overline{\overline{\nabla_{X} Y}}-\frac{1}{2} p^{*}(\omega(X, Y)) E-p^{*}(\hat{s}(X, Y)) \partial_{s} \\
\nabla_{E}^{P} \overline{\bar{X}} & =\nabla_{\overline{\bar{X}}}^{P} E=2 \overline{\overline{\sigma X}}+p^{*}(\omega(X, u)) \partial_{s} \\
\nabla_{\partial_{s}}^{P} \overline{\bar{X}} & =\nabla_{\overline{\bar{X}}}^{P} \partial_{s}=\overline{\bar{X}} \\
\nabla_{E}^{P} E & =p^{*} f \partial_{s}-2 \overline{\bar{U}} \\
\nabla_{E}^{P} \partial_{s} & =\nabla_{\partial_{s}}^{P} E=E \\
\nabla_{\partial_{s}}^{P} \partial_{s} & =\partial_{s}
\end{aligned}
$$

where $f$ is a function on $M, U$ is a vector field on $M, \hat{s}$ is a symmetric 2-tensor on $M$, and $\sigma$ is the endomorphism of $T M$ associated to $s$, hence $\hat{s}(X, Y)=\omega(X, \sigma Y)$.

These formulas have the correct linearity properties and yield a torsion free linear connection on $P$. One checks readily that $\nabla^{P} \mu=0$ so that $\nabla^{P}$ is a symplectic connection on $(P, \mu)$.

We now compute the curvature $R^{\nabla^{P}}$ of this connection $\nabla^{P}$. We get

$$
\begin{aligned}
& \begin{aligned}
R^{\nabla^{P}}(\overline{\bar{X}}, \overline{\bar{Y}}) \overline{\bar{Z}}= & \overline{\overline{R^{\nabla}(X, Y) Z}} \\
& +\overline{\overline{2 \omega(X, Y) \sigma Z-\omega(Y, Z) \sigma X+\omega(X, Z) \sigma Y-\hat{s}(Y, Z) X+\hat{s}(X, Z) Y}}
\end{aligned} \\
& +p^{*}\left[\omega(X, D(\sigma, U)(Y, Z))-\omega(Y, D(\sigma, U)(X, Z)] \partial_{s}\right. \\
& R^{\nabla^{P}}(\overline{\bar{X}}, \overline{\bar{Y}}) E=\overline{\overline{2 D(\sigma, U)(X, Y)-2 D(\sigma, U)(Y, X)}} \\
& +p^{*}\left[\omega\left(X, \frac{1}{2} f Y-\nabla_{Y} U-2 \sigma^{2} Y\right)-\omega\left(Y, \frac{1}{2} f X-\nabla_{X} U-2 \sigma^{2} X\right)\right] \partial_{s} \\
& R^{\nabla^{P}}(\overline{\bar{X}}, E) \overline{\bar{Y}}=\overline{\overline{2 D(\sigma, U)(X, Y)}}-p^{*}\left[\omega\left(Y, \frac{1}{2} f X-\nabla_{X} U-2 \sigma^{2} X\right)\right] \partial_{s} \\
& R^{\nabla^{P}}(\overline{\bar{X}}, E) E=2 \overline{\overline{\frac{1}{2} f X-\nabla_{X} U-2 \sigma^{2} X}}+p^{*}[X f+4 s(X, u)] \partial_{s} \\
& R^{\nabla^{P}}(\overline{\bar{X}}, \overline{\bar{Y}}) \partial_{s}=0 \quad R^{\nabla^{P}}(\overline{\bar{X}}, E) \partial_{s}=0 \\
& R^{\nabla^{P}}\left(\overline{\bar{X}}, \partial_{s}\right) \overline{\bar{Y}}=0 \quad R^{\nabla^{P}}\left(\overline{\bar{X}}, \partial_{s}\right) E=0 \quad R^{\nabla^{P}}\left(\overline{\bar{X}}, \partial_{s}\right) \partial_{s}=0 \\
& R^{\nabla^{P}}\left(E, \partial_{s}\right) \overline{\bar{X}}=0 \quad R^{\nabla^{P}}\left(E, \partial_{s}\right) E=0 \quad R^{\nabla^{P}}\left(E, \partial_{s}\right) \partial_{s}=0
\end{aligned}
$$

where

$$
D(\sigma, U)\left(Y, Y^{\prime}\right):=\left(\nabla_{Y} \sigma\right) Y^{\prime}+\frac{1}{2} \omega\left(Y^{\prime}, U\right) Y-\frac{1}{2} \omega\left(Y, Y^{\prime}\right) U .
$$

The Ricci tensor $r^{\nabla^{P}}$ of the connection $\nabla^{P}$ is given by

$$
\begin{aligned}
r^{\nabla^{P}}(\overline{\bar{X}}, \overline{\bar{Y}}) & =r^{\nabla}(X, Y)+2(n+1) \hat{s}(X, Y) \\
r^{\nabla^{P}}(\overline{\bar{X}}, E) & =-(2 n+1) \omega(X, u)-2 \operatorname{Tr}\left[Y \rightarrow\left(\nabla_{Y} \sigma\right)(X)\right] \\
r^{\nabla^{P}}\left(\overline{\bar{X}}, \partial_{s}\right) & =0 \\
r^{\nabla^{P}}(E, E) & =4 \operatorname{Tr}\left(\sigma^{2}\right)-2 n f+2 \operatorname{Tr}\left[X \rightarrow \nabla_{X} U\right] \\
r^{\nabla^{P}}\left(E, \partial_{s}\right) & =0 \\
r^{\nabla^{P}}\left(\partial_{s}, \partial_{s}\right) & =0
\end{aligned}
$$

Theorem 7.4 [17] In the framework described above, $\nabla^{P}$ is a symplectic connection on $(P, \mu)$ for any choice of $\hat{s}, U$ and $f$. The vector field $E$ on $P$ is affine $\left(L_{\tilde{E}} \nabla^{P}=0\right)$ and symplectic $\left(L_{\tilde{E}} \mu=0\right)$; the vector field $\partial_{s}$ on $P$ is affine and conformal $\left(L_{\partial_{s}} \mu=2 \mu\right)$. 
Furthermore, choosing

$$
\begin{aligned}
\hat{s} & =\frac{-1}{2(n+1)} r^{\nabla} \\
\underline{U}: & =\omega(U, \cdot)=\frac{2}{2 n+1} \operatorname{Tr}\left[Y \rightarrow \nabla_{Y} \sigma\right] \\
f & =\frac{1}{2 n(n+1)^{2}} \operatorname{Tr}\left(\rho^{\nabla}\right)^{2}+\frac{1}{n} \operatorname{Tr}\left[X \rightarrow \nabla_{X} U\right] .
\end{aligned}
$$

we have:

- the connection $\nabla^{P}$ on $(P, \mu)$ is Ricci-flat (i.e. has zero Ricci tensor);

- if the symplectic connection $\nabla$ on $(M, \omega)$ is of Ricci-type, then the connection $\nabla^{P}$ on $(P, \mu)$ is flat.

- if the connection $\nabla^{P}$ is locally symmetric, the connection $\nabla$ is of Ricci-type, hence $\nabla^{P}$ is flat.

Proof The first point is an immediate consequences of the formulas above for $r^{\nabla^{P}}$. The second point is a consequence of the differential identities satisfied by the Ricci-type symplectic connections. The third point comes from the fact that $\left(\nabla_{\overline{\bar{Z}}}^{P} R^{\nabla^{P}}\right)(\overline{\bar{X}}, \overline{\bar{Y}}) \overline{\bar{T}}$ contains only one term in $E$ whose coefficient is $\frac{1}{2} W^{\nabla^{P}}(X, Y, T, Z)$.

\subsection{Examples of contact quadruples}

We give here examples of contact quadruples corresponding to a given symplectic manifold $(M, \omega)$ (i.e. examples of $(N, \alpha, \pi)$ where $N$ is a smooth $2 n+1$ dimensional manifold, $\alpha$ is a 1 -form on $N$ such that $\alpha \wedge(d \alpha)^{n}$ is nowhere vanishing, $\pi: N \rightarrow M$ is a smooth submersion and $d \alpha=\pi^{*} \omega$.

- Let $(M, \omega=d \lambda)$ be an exact symplectic manifold. Define $N=M \times \mathbb{R}, \pi=p_{1}$ (=projection of the first factor), $\alpha=d t+p_{1}^{*} \lambda$; then $(N, \alpha)$ is a contact manifold and $(M, N, \alpha, \pi)$ is a contact quadruple.

The associated induced manifold is $P=N \times \mathbb{R}=M \times \mathbb{R}^{2}$; with coordinates $(t, s)$ on $\mathbb{R}^{2}$ and obvious identification

$$
\mu=\mathrm{e}^{2 s}[d \lambda+2 d s \wedge(d t+\lambda)]
$$

- Let $(M, \omega)$ be a quantizable symplectic manifold; this means that there is a complex line bundle $L \stackrel{p}{\longrightarrow} M$ with hermitean structure $h$ and a connection $\nabla$ on $L$ preserving $h$ whose curvature is proportional to $i \omega$.

Define $N:=\{\xi \in L \mid h(\xi, \xi)=1\} \subset L$ to be the unit circle sub-bundle. It is a principal $U(1)$ bundle and $L$ is the associated bundle $L=N \times_{U(1)} \mathbb{C}$. The connection 1-form on $N$ (representing $\nabla)$ is $u(1)=i \mathbb{R}$ valued and will be denoted $\alpha^{\prime}$; its curvature is $d \alpha^{\prime}=i k \omega$. Define $\alpha:=\frac{1}{i k} \alpha^{\prime}$ and $\pi:=\left.p\right|_{N}: N \rightarrow M$ the surjective submersion. Then $(M, N, \alpha, \pi)$ is a contact quadruple.

The associated induced manifold $P$ is in bijection with $L_{0}=L \backslash$ zero section.

- Let $(M, \omega)$ be a connected homogeneous symplectic manifold; i. e. $M=G / H$ where $G$ is a Lie group which we may assume connected and simply connected and where $H$ is the stabilizer in $G$ of a point $x_{0} \in M$. If $p: G \rightarrow M: g \rightarrow g x_{0}, \quad p^{*} \omega$ is a left invariant closed 2-form on $G$ and $\Omega=\left(p^{*} \omega\right)_{e},(\mathrm{e}=$ neutral element of $G)$ is a Chevalley 2-cocycle on $\mathfrak{g}(=$ Lie Algebra of $G)$ with 
values in $\mathbb{R}$ (for the trivial representation).

Notice that $\Omega$ vanishes as soon as one of its arguments is in $\mathfrak{h}(=$ Lie algebra of $H)$. Let $\mathfrak{g}_{1}=\mathfrak{g} \oplus \mathbb{R}$ be the central extension of $\mathfrak{g}$ defined by $\Omega$; i. e.

$$
[(X, a),(Y, b)]=([X, Y], \Omega(X, Y)) .
$$

Let $\mathfrak{h}^{\prime}$ be the subalgebra of $\mathfrak{g}_{1}$, isomorphic to $\mathfrak{h}$, defined by $\mathfrak{h}^{\prime}:=\{(X, 0) \mid X \in \mathfrak{h}\}$. Let $G_{1}$ be the connected and simply connected group of algebra $\mathfrak{g}$, and let $H^{\prime}$ be the connected subgroup of $G_{1}$ with Lie algebra $\mathfrak{h}^{\prime}$. Assume $H^{\prime}$ is closed.

Then $G_{1} / H^{\prime}$ admits a natural structure of smooth manifold; define $N:=G_{1} / H^{\prime}$. Let $p_{1}: G_{1} \rightarrow G$ be the homomorphism whose differential is the projection $\mathfrak{g}_{1} \rightarrow \mathfrak{g}$ on the first factor; clearly $p_{1}\left(H^{\prime}\right) \subset H$. Define $\pi: N=G_{1} / H^{\prime} \rightarrow M=G / H: g_{1} H^{\prime} \mapsto p_{1}\left(g_{1}\right) H$; it is a surjective submersion.

The contact form $\alpha$ on $N$ is constructed as follows: $p_{1}^{*} \circ p^{*} \omega$ is a left invariant closed 2-form on $G_{1}$ vanishing on the fibres of $p \circ p_{1}: G_{1} \rightarrow M$. Its value $\Omega_{1}$ at the neutral element $e_{1}$ of $G_{1}$ is a Chevalley 2-cocycle of $\mathfrak{g}_{1}$ with values in $\mathbb{R}$. Define the 1-cochain $\alpha_{1}: \mathfrak{g}_{1} \rightarrow \mathbb{R}:(X, a) \rightarrow-a$. Then $\Omega_{1}=\delta \alpha_{1}$ is a coboundary. Let $\tilde{\alpha}_{1}$ be the left invariant 1-form on $G_{1}$ corresponding to $\alpha_{1}$. Let $q: G_{1} \rightarrow G_{1} / H^{\prime}=N$ be the natural projection.

There exists a 1 -form $\alpha$ on $N$ so that $q^{*} \alpha=\tilde{\alpha}_{1}$. Indeed, for any $X \in \mathfrak{h}^{\prime}$ we have

$$
\begin{aligned}
& i(\tilde{X}) \tilde{\alpha}_{1}=\alpha_{1}(X)=0 \\
& \left.\left.\left(L_{\tilde{X}} \tilde{\alpha}_{1}\right)(\widetilde{(} Y, b)\right)=-\tilde{\alpha}_{1}([\tilde{X}, \tilde{(} Y, b)]\right)=-\alpha_{1}([X,(Y, b)])=\Omega(X, Y)=0
\end{aligned}
$$

where $\tilde{U}$ is the left invariant vector field on $G_{1}$ corresponding to $U \in \mathfrak{g}_{1}$. Furthermore $d \alpha=\pi^{*} \omega$ because both are $G_{1}$ invariant 2-forms on $N$ and:

$$
\begin{aligned}
(d \alpha)_{q\left(e_{1}\right)}\left((X, a)^{* N},(Y, b)^{* N}\right) & =\left(q^{*} d \alpha\right)_{e_{1}}(\widetilde{(X, a)}, \widetilde{(Y, b)}) \\
& =\left(d \tilde{\alpha}_{1}\right)_{e_{1}}(\widetilde{(X, a)}, \widetilde{(Y, b)}) \\
& =\Omega(X, Y) \\
& =\omega_{x_{0}}\left(X^{* M}, Y^{* M}\right) \\
& =\left(\pi^{*} \omega\right)_{q\left(e_{1}\right)}\left((X, a)^{* N},(Y, b)^{* N}\right)
\end{aligned}
$$

where we denote by $U^{* N}$ the fundamental vector field on $N$ associated to $U \in \mathfrak{g}_{1}$.

- If $(M, \omega, \nabla)$ is a simply connected symplectic manifold with a Ricci-type connection we have seen in Section 4.4 how to build the manifold $N$ as a holonomy bundle over $M$ corresponding to a connection built on the extension $B^{\prime}(M)$ of the frame bundle $B(M)$ over $M$.

\subsection{More about reduction}

Let $\left(P, \omega^{P}\right)$ be a symplectic manifold of dimension $(2 n+2)$. Assume $P$ admits a conformal vector field $S$ :

$$
L_{S} \omega^{P}=2 \omega^{P} ; \quad \text { define } \alpha:=\frac{1}{2} i(S) \omega^{P} \quad \text { so that } d \alpha=\omega^{P} .
$$

Assume also that $P$ admits a symplectic vector field $\tilde{E}$ commuting with $S$

$$
L_{\tilde{E}} \omega^{P}=0 \quad[S, \tilde{E}]=0 \quad\left(\Rightarrow L_{\tilde{E}} \alpha=0\right) .
$$


Define

$$
\Sigma=\left\{x \in P \mid \omega_{x}^{P}(S, \tilde{E})=1\right\}
$$

and assume that it is non-empty. The tangent space to the hypersurface $\Sigma$ is given by

$$
T_{x} \Sigma=\operatorname{ker}\left(i(\tilde{E}) \omega^{P}\right)_{x}=\tilde{E}^{\perp_{\omega} P} .
$$

The restriction of $\omega_{x}^{P}$ to $T_{x} \Sigma$ has rank $2 n-2$ and a radical spanned by $\tilde{E}_{x}$.

The restriction of $\alpha$ to $\Sigma$ is a contact 1 -form on $\Sigma$.

Let $\sim$ be the equivalence relation defined on $\Sigma$ by the flow of $\tilde{E}$. Assume that the quotient $\Sigma / \sim$ has a $2 n$ dimensional manifold $M$ structure so that $\pi: \Sigma \rightarrow \Sigma / \sim=M$ is a smooth submersion. Define on $\Sigma$ a "horizontal" distribution of dimension $2 n, \mathscr{H}$, by

$$
\mathscr{H}=>\tilde{E}, S<{ }^{\perp}{ }_{\omega} P,
$$

and remark that $\pi_{* \mid \mathscr{H}_{y}}: \mathscr{H}_{y} \rightarrow T_{x=\pi(y)} M$ is an isomorphism.

Define as usual the reduced 2-form $\omega^{M}$ on $M$ by

$$
\omega_{x=\pi(y)}^{M}\left(Y_{1}, Y_{2}\right)=\omega_{y}^{P}\left(\bar{Y}_{1}, \bar{Y}_{2}\right)
$$

where $\bar{Y}_{i}(i=1,2)$ is defined by (i) $\pi_{*} \bar{Y}_{i}=Y_{i}$ (ii) $\bar{Y}_{i} \in \mathscr{H}_{y}$.

Notice that $\pi_{*}[\tilde{E}, \bar{Y}]=0$, and $\omega^{P}(S,[\tilde{E}, \bar{Y}])=-L_{\tilde{E}} \omega^{P}(S, \bar{Y})+\tilde{E} \omega^{P}(S, \bar{Y})=0$ hence

$$
[\tilde{E}, \bar{Y}]=0 .
$$

The definition of $\omega_{x}^{M}$ does not depend on the choice of $y$. Indeed

$$
\tilde{E} \omega^{P}\left(\bar{Y}_{1}, \bar{Y}_{2}\right)=L_{\tilde{E}} \omega^{P}\left(\bar{Y}_{1}, \bar{Y}_{2}\right)+\omega^{P}\left(\left[\tilde{E}, \bar{Y}_{1}\right], \bar{Y}_{2}\right)+\omega^{P}\left(\bar{Y}_{1},\left[\tilde{E}, \bar{Y}_{2}\right]\right)=0 .
$$

Clearly $\omega^{M}$ is of maximal rank $2 n$ as $\mathscr{H}$ is a symplectic subspace. Finally

$$
\begin{aligned}
\pi^{*}\left(d \omega^{M}\left(Y_{1}, Y_{2}, Y_{3}\right)\right) & =\underset{123}{\circlearrowright}\left(Y_{1} \omega^{M}\left(Y_{2}, Y_{3}\right)-\omega^{M}\left(\left[Y_{1}, Y_{2}\right], Y_{3}\right)\right) \\
& =\underset{123}{\bigoplus}\left(\bar{Y}_{1} \omega^{P}\left(\bar{Y}_{2}, \bar{Y}_{3}\right)-\omega^{P}\left(\overline{\left[Y_{1}, Y_{2}\right]}, \bar{Y}_{3}\right)\right)
\end{aligned}
$$

and

$$
\left[\bar{Y}_{1}, \bar{Y}_{2}\right]=\overline{\left[Y_{1}, Y_{2}\right]}+\omega^{P}\left(S,\left[\bar{Y}_{1}, \bar{Y}_{2}\right]\right) \tilde{E} .
$$

Hence $\omega^{M}$ is closed and thus symplectic. Clearly $\pi^{*} \omega^{M}=\omega_{\mid \Sigma}^{P}=d\left(\alpha_{\mid \Sigma}\right)$.

Remark 7.5 The manifold $M$ is the first element of a contact quadruple $\left(M, \Sigma, \frac{1}{2} \alpha_{\left.\right|_{\Sigma}}, \pi\right)$.

We shall now consider the reduction of a connection. Let $\left(P, \omega^{P}\right), \tilde{E}, S, \Sigma, M, \omega^{M}$ be as above. Let $\nabla^{P}$ be a symplectic connection on $P$ and assume that the vector field $\tilde{E}$ is affine $\left(L_{\tilde{E}} \nabla^{P}=0\right)$.

Then define a connection $\nabla^{\Sigma}$ on $\Sigma$ by

$$
\nabla_{A}^{\Sigma} B:=\nabla_{A}^{P} B-\omega^{P}\left(\nabla_{A}^{P} B, \tilde{E}\right) S=\nabla_{A}^{P} B+\omega^{P}\left(B, \nabla_{A}^{P} \tilde{E}\right) S .
$$

Then $\nabla^{\Sigma}$ is a torsion free connection and $\tilde{E}$ is an affine vector field for $\nabla^{\Sigma}$.

Define a connection $\nabla^{M}$ on $M$ by:

$$
\overline{\nabla_{Y_{1}}^{M} Y_{2}}(y)=\nabla_{\bar{Y}_{1}}^{\Sigma} \bar{Y}_{2}(y)-\omega^{P}\left(\bar{Y}_{2}, \nabla_{\bar{Y}_{1}}^{P} S\right) \tilde{E} .
$$

If $x \in M$, this definition does not depend on the choice of $y \in \pi^{-1}(x)$ and one can check that the connection $\nabla^{M}$ is symplectic. 
Lemma 7.6 [17] Let $\left(P, \omega^{P}\right)$ be a symplectic manifold admitting a symplectic connection $\nabla^{P}$, a conformal vector field $S$, a symplectic vector field $\tilde{E}$ which is affine and commutes with $S$. If the constraint manifold $\Sigma=\left\{x \in P \mid \omega_{x}(S, \tilde{E})=1\right\}$ is not empty, and if the reduction of $\Sigma$ is a manifold $M$, this manifold admits a symplectic structure $\omega^{M}$ and a natural reduced symplectic connection $\nabla^{M}$.

In particular

Theorem 7.7 [17] Let $\left(P, \omega^{P}\right)$ be a symplectic manifold admitting a conformal vector field $S\left(L_{S} \mu=\right.$ $2 \mu)$ which is complete, a symplectic vector field $\tilde{E}$ which commutes with $S$ and assume that, for any $x \in P, \mu_{x}(S, \tilde{E})>0$. If the reduction of $\Sigma=\left\{x \in P \mid \mu_{x}(S, \tilde{E})=1\right\}$ by the flow of $\tilde{E}$ has a manifold structure $M$ with $\pi: \Sigma \rightarrow M$ a surjective submersion, then $M$ admits a reduced symplectic structure $\omega^{M}$ and $\left(P, \omega^{P}\right)$ is obtained by induction from $\left(M, \omega^{M}\right)$ using the contact quadruple $\left(M, \Sigma, \frac{1}{2} i(S) \omega_{\left.\right|_{\Sigma}}^{P}, \pi\right)$.

In particular $\left(P, \omega^{P}\right)$ admits a Ricci-flat connection.

\section{Non-commutative symplectic symmetric spaces}

\subsection{Motivations}

After the celebrated example of the quantum torus and related non-commutative spaces [39, 24], it appeared natural to try to define non-commutative spaces through oscillatory integral formulae in the same spirit of [39] but with larger symmetry groups — other than $\mathbb{R}^{d}$ — implementing this way in our class of non-commutative manifolds not only an operator algebraic framework but also a strong geometric content.

In the context of symmetric spaces, this leads to the so-called 'WKB-quantisation of symplectic symmetric spaces' as introduced by Karasëv, Weinstein and Zakrzewski in the mid 90's (see [47] and references therein). Originally (cf. [47]), the following question was raised in the framework of Hermitean symmetric spaces of the non-compact type $M=G / K$.

Question 8.1 Given a $G$-invariant product on $M$ expressed in the 'WKB form':

$$
\left(u \star_{\theta} v\right)(x)=\frac{1}{\theta^{2 n}} \int_{M \times M} a_{\theta}(x, y, z) \exp \left(\frac{i}{\theta} S(x, y, z)\right) u(y) v(z) d y d z,
$$

which conditions on the phase function $S \in C^{\infty}\left(M^{3}, \mathbb{R}\right)$ and on the amplitude $a_{\theta}=a_{0}+\theta a_{1}+\theta^{2} a_{2}+\ldots \in$ $C^{\infty}\left(M^{3}, \mathbb{R}\right)[[\theta]]$ does one need in order to guarantee formal associativity of $\star_{\theta}$ ?

Weinstein provided some evidence showing that the phase function $S$ should be closely related to the three point function denoted hereafter $S_{\mathrm{can}}$ and (partially) defined as follows. Given three points $x, y$ and $z$ in the symmetric space with the property that the equation $s_{x} s_{y} s_{z}(X)=X$ admits a (unique) solution $X$, the value of $S_{\text {can }}(x, y, z)$ is given by the symplectic area

$$
S_{\mathrm{can}}(x, y, z)=\int_{X Y \dot{Y} Z} \omega
$$

where $X \stackrel{\Delta}{Y} Z$ denotes the geodesic triangle with vertices

$$
X, \quad Y:=s_{z}(X), \quad Z=s_{y} s_{z}(X) .
$$


The question of characterising geometrically the amplitude was left open.

Another important aspect in this problematic is to determine whether such a WKB product underlies topological function algebras analogous to the continuous field of $C^{\star}$-algebras deforming $C(\mathbb{T})$ in the case of the quantum torus $\mathbb{T}_{\theta}$. More precisely:

Question 8.2 Given $\theta$ in some deformation parameter space, does one have a function space $\mathbb{A}_{\theta}$

$$
C_{c}^{\infty}(M) \subset \mathbb{A}_{\theta} \subset \mathscr{D}^{\prime}(M)
$$

such that the pair $\left(\mathbb{A}_{\theta}, \star_{\theta}\right)$ is a topological $G$-algebra ${ }^{1}$ ?

In the present section, we survey a geometrical approach to these questions initiated in [5] and based on the definition of a class of three point functions, called hereafter 'admissible', on symplectic symmetric spaces. Admissible functions are characterised by compatibility properties with the symmetries of the symmetric space at hand. We will show how these properties guarantee associativity of the oscillating product associated to such an admissible function in the case of a cocyclic function. We will then indicate on a curved two dimensional example (a generic coadjoint orbit of the Poincaré group in dimension 1+1) how the cocycle condition can be relaxed by introducing a non-trivial amplitude in the oscillating kernel. We will end by mentioning a result which solves Question 8.2 above in this particular context. Let us add that the general results obtained in this direction go far beyond the particular example presented here. For instance, the solution for the general solvable symmetric case has led to several universal deformation formulae for actions of various classes of solvable Lie groups [10, 9, 6]. Applications in noncommutative geometry (non-commutative causal black holes [7]) as well as in analytic number theory (Rankin-Cohen brackets on modular forms [11]) have been developed.

\subsection{Basic definitions and the cocyclic case}

Denoting by $K:=a_{\theta} e^{\frac{i}{\theta} S}$ the oscillating kernel defining the product given in (30), a simple computation shows that associativity of $\star_{\theta}$ is (at least formally) equivalent to the following condition:

$$
\int_{M} K(a, b, t) K(t, c, d) \mu(t)=\int_{M} K(a, \tau, d) K(\tau, b, c) \mu(\tau)
$$

for every quadruple of points $a, b, c, d$ in $M$. Equation (31) obviously holds if one can pass from one integrand to the other using a change of variables $\tau=\varphi(t)$. This motivates

Definition 8.3 Let $(M, \mu)$ be an orientable manifold endowed with a volume form $\mu$. A three-point kernel $K \in C^{\infty}(M \times M \times M)$ is geometrically associative if for every quadruple of points $a, b, c, d$ in $M$ there exists a volume preserving diffeomorphism

$$
\varphi:(M, \mu) \rightarrow(M, \mu)
$$

such that for all $t$ in $M$ :

$$
K(a, b, t) K(t, c, d)=K(a, \varphi(t), d) K(\varphi(t), b, c) .
$$

\footnotetext{
${ }^{1}$ that is, an associative algebra underlying a topological vector space on which the group $G$ acts strongly continuously by algebra automorphisms.
} 
In the sequel, we give sufficient conditions for geometric associativity. From now on, $(M, \omega, s)$ denotes a symplectic symmetric space (not necessarily Hermitean). We first observe the following group-like cohomological complex naturally associated to our context.

Definition 8.4 A $k$-cochain on $M$ is a totally skew symmetric real-valued smooth function $S$ on $M^{k}$ which is invariant under the (diagonal) action of the symmetries $\left\{s_{x}\right\}_{x \in M}$. Denoting by $P^{k}(M)$, the space of $k$-cochains, one has the cohomology operator $\delta: P^{k}(M) \rightarrow P^{k+1}(M)$ defined as

$$
(\delta S)\left(x_{0}, \ldots, x_{k}\right):=\sum_{j}(-1)^{j} S\left(x_{0}, \ldots, \hat{x_{j}}, \ldots, x_{k}\right) .
$$

Definition 8.5 A 3-cochain $S \in P^{3}(M)$ is called admissible if for all $x \in M$, one has

$$
S(x, y, z)=-S\left(x, s_{x}(y), z\right) \quad \forall y, z \in M
$$

A Weyl triple is the data of a symmetric space $M$ endowed with an invariant volume form $\mu$ together with the data of an admissible 3 -cocycle $S$ (i.e. $\delta S=0$ ).

Skew-symmetry naturally leads us to adopt the following "oriented graph" type notation for a 3-cochain $S:$

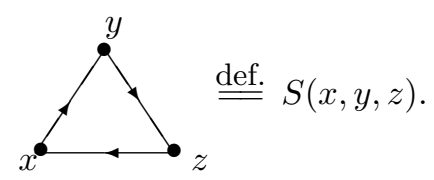

A change of orientation in such an "oriented triangle" leads to a change of sign of its value. However, the value represented by such a "triangle" does not depend on the way it "stands", only the data of the vertices and the orientation of the edges matters.

Now, consider a Weyl triple $(M, \mu, S)$, and let $A$ be some (topological) associative algebra. And, for compactly supported functions $u$ and $v \in C_{c}^{\infty}(M, A)$, consider the following "product":

$$
u \star v(x)=\int_{M \times M} u(y) v(z) e^{i S(x, y, z)} \mu(y) \mu(z) .
$$

With the above notation for $S$, associativity for $\star$ now formally takes the following form:

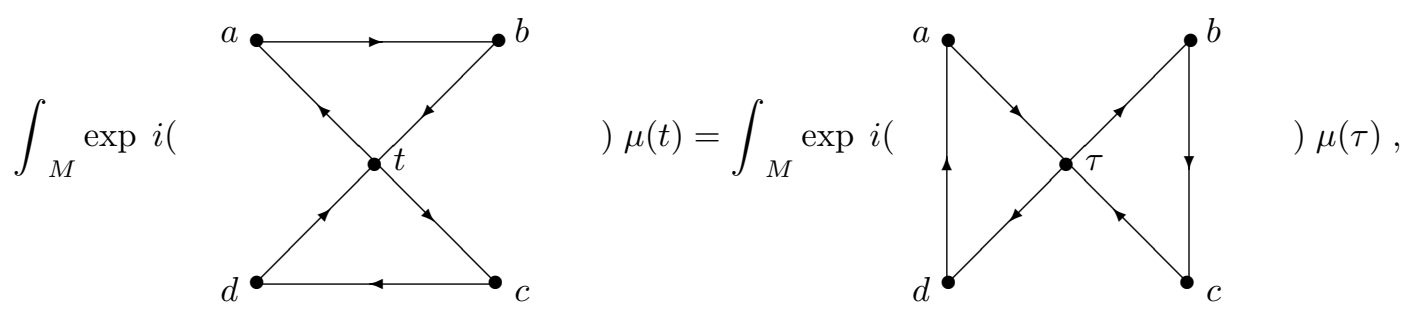

for every quadruple of points $a, b, c, d$ in $M$.

In the above formula, the diagram in the argument of the exponential in the LHS (respectively the RHS) stands for $S(a, b, t)+S(t, c, d)$ (respectively $S(a, d, \tau)+S(\tau, b, c)$ ). 
Proposition 8.6 Let $(M, \mu, S)$ be a Weyl triple. Then, the associated three-point kernel $K=e^{i S}$ is geometrically associative.

Proof. Fix four points $a, b, c, d$. Regarding Definition 8.3 and formula (33), one needs to construct our volume preserving diffeomorphism $\varphi:(M, \mu) \rightarrow(M, \mu)$ in such a way that for all $t$,
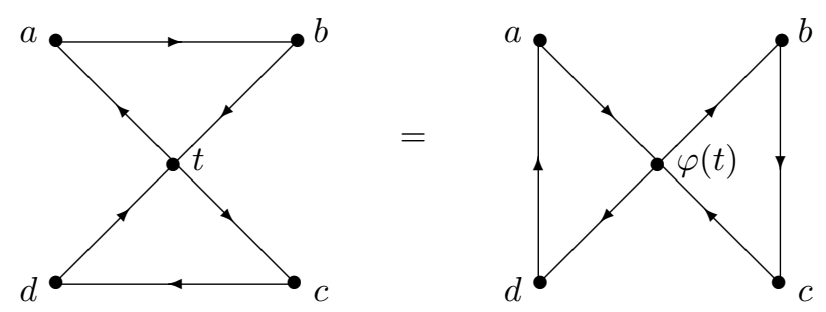

We first observe that the data of four points $a, b, c, d$ determines what we call an " $S$-barycentre", that is a point $g=g(a, b, c, d)$ such that
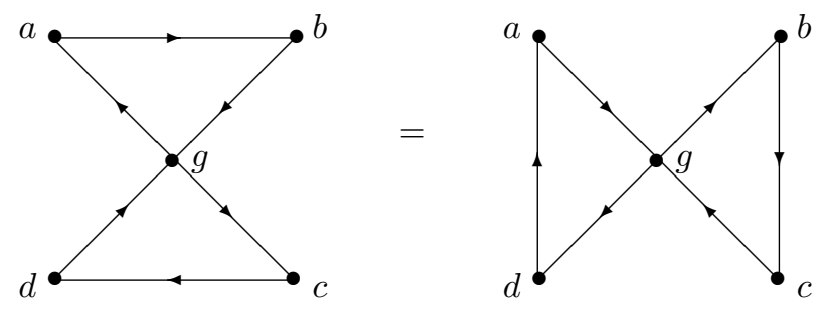

Indeed, since
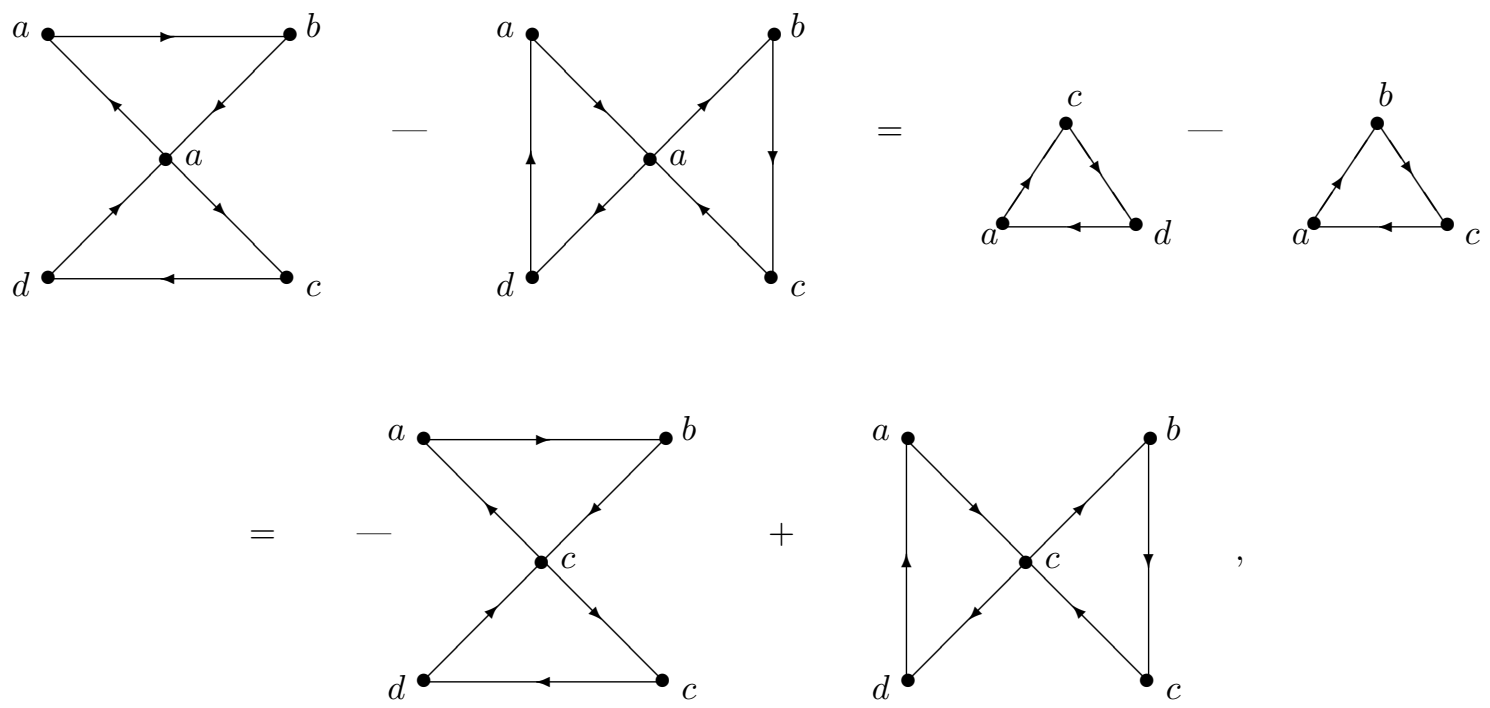

any continuous path joining $a$ to $c$ contains such a point $g$. 
Now, we fix once for all such an $S$-barycentre $g$ for $\{a, b, c, d\}$ and we adopt the following notation. For all $x$ and $y$ in $M$, the value of $S(g, x, y)$ is denoted by a "thickened arrow":

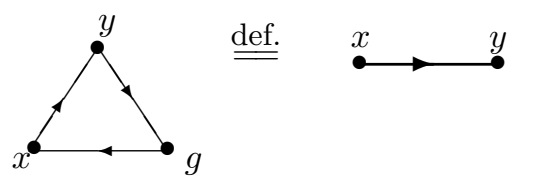

Again, a change of orientation in such an arrow changes the sign of its value. Also, admissibility which has the form
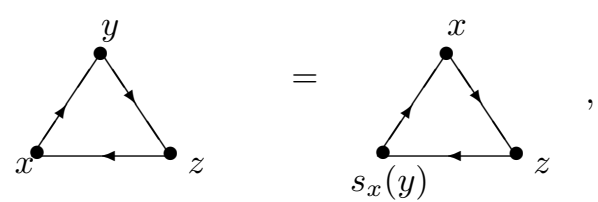

implies

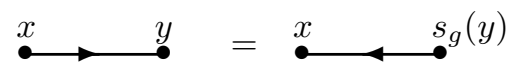

for all $x$ and $y$ in $M$. While, from cocyclicity, one gets

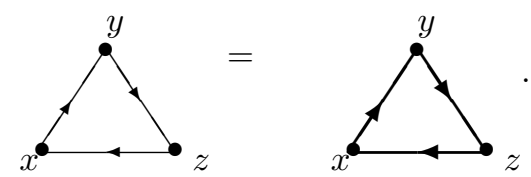

Moreover, the barycentric property of $g$ can be written
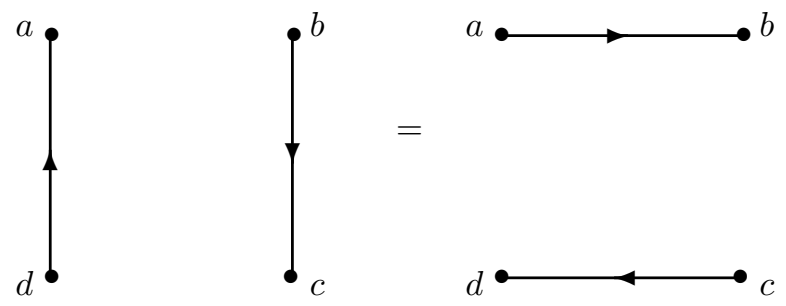

$=$

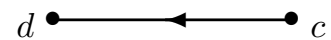


Hence
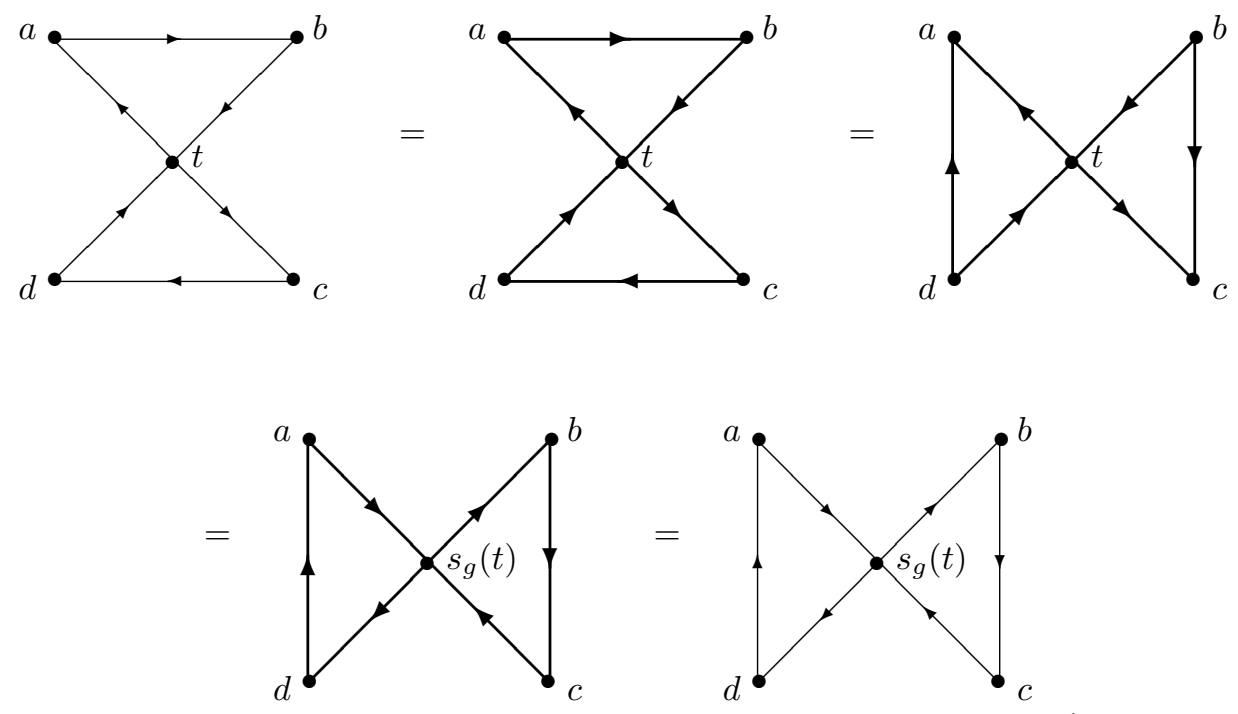

One can therefore choose our diffeomorphism $\varphi$ as

$$
\varphi=s_{g}
$$

Remark 8.7 Given a symplectic symmetric space Weinstein's function $S_{\text {can }}$ turns out to be admissible (wherever it's well-defined) [5]. However, the curvature is the obstruction to the cocyclicity of $S_{\text {can }}$.

\subsection{A curved example: $S O(1,1) \times \mathbb{R}^{2} / \mathbb{R}$}

In what follows, we analyse in some details the case of the solvable symplectic symmetric surface $M=S O(1,1) \times \mathbb{R}^{2} / \mathbb{R}$. As a homogeneous symplectic manifold it can be realised as a generic coadjoint orbit of the Poincaré group $G=S O(1,1) \times \mathbb{R}^{2}$. In the dual $\mathfrak{g}^{\star}$ of Lie algebra $\mathfrak{g}$ of $G$, the orbit $M$ sits as hyperbolic cylinder. In this picture, the geodesics of the canonical symplectic symmetric connection $\nabla$ are planar sections of $M \subset \mathbb{R}^{3}$. The affine manifold $(M, \nabla)$ turns out to be strictly geodesically convex. Moreover, given three points $x, y, z$ in $M$, the equation $s_{x} s_{y} s_{z}(t)=t$ has always a (unique) solution $t \in M$. In particular, Weinstein's function $S_{\text {can }}$ is everywhere defined on $M \times M \times M$. Within suitable global Darboux coordinates $(M, \omega) \simeq\left(\mathbb{R}^{2}, d a \wedge d \ell\right)$, the symmetry at $(a, \ell)$ has the following expression:

$$
s_{(a, \ell)}\left(a^{\prime}, \ell^{\prime}\right)=\left(2 a-a^{\prime}, 2 \cosh \left(a-a^{\prime}\right) \ell-\ell^{\prime}\right)
$$

while Weinstein's function is given by

$$
S_{\text {can }}\left(\left(a_{1}, \ell_{1}\right),\left(a_{2}, \ell_{2}\right),\left(a_{3}, \ell_{3}\right)\right)=\underset{1,2,3}{(} \sinh \left(a_{1}-a_{2}\right) \ell_{3}
$$

Observe that in this coordinate system one sees how far $M$ is from being flat: indeed replacing, in the expression of the symmetry map, the function

$$
A^{0}: M \times M \rightarrow \mathbb{R}:\left((a, \ell),\left(a^{\prime}, \ell^{\prime}\right)\right) \mapsto \cosh \left(a-a^{\prime}\right)
$$


by the constant function 1 would yield the flat plane. This function turns out to be exactly the one which twists the volume form $\omega \wedge \omega$ on $M \times M$ in the expression of a WKB quantisation product. More precisely, one has

Theorem 8.8 [5] There exist Fréchet function spaces $\left\{\mathscr{A}_{\theta}\right\}_{\theta \in \mathbb{R}}$ such that

(i) for all $\theta$, one has

$$
C_{c}^{\infty}(M) \subset \mathscr{A}_{\theta} \subset C_{\infty}(M)
$$

(ii) the formula

$$
\left(u \star_{\theta} v\right)(x)=\frac{1}{\theta^{2}} \int_{M \times M} A^{0}(y, z) \exp \left(\frac{i}{\theta} S_{\text {can }}(x, y, z)\right) u(y) v(z) d y d z
$$

defines an associative product on $\mathscr{A}_{\theta} \quad \theta \neq 0$. Each pair $\left(\mathscr{A}_{\theta}, \star_{\theta}\right)$ is then a Fréchet algebra.

(iii) For $u$ and $v$ smooth compactly supported functions on $M$, one has an asymptotic expansion in powers of $\theta$ :

$$
u \star_{\theta} v \sim u v+\frac{\theta}{2 i}\{u, v\}+\text { higher order terms }
$$

where $\{$,$\} denotes the Poisson structure associated to the symplectic form \omega$.

An analysis of the $G$-equivariant (formal) equivalences yields a WKB expression for every star product on $M$ from the data of the preceding one. More precisely, one has

Proposition 8.9 [8] Let $\mathscr{P} \in C^{\infty}(\mathbb{R})[[\theta]]$ be a formal function on $\mathbb{R}$. Then, an asymptotic expansion in powers of $\theta$ of the following oscillatory integral

$$
\left(u \star_{\theta}^{\mathscr{P}} v\right)(x)=\frac{1}{\theta^{2}} \int_{M \times M} \frac{\mathscr{P}\left(a_{\mathbf{x}}-a_{\mathbf{z}}\right) \mathscr{P}\left(a_{\mathbf{y}}-a_{\mathbf{x}}\right)}{\mathscr{P}\left(a_{\mathbf{y}}-a_{\mathbf{z}}\right)} A^{0}(y, z) \exp \left(\frac{i}{\theta} S_{\mathrm{can}}(x, y, z)\right) u(y) v(z) d y d z
$$

yields a G-invariant star product on $M$. Moreover, every $G$-invariant star product on $M$ may be described this way. The choice

$$
\mathscr{P}(a)=\sqrt{\cosh (a)}
$$

yields a strongly closed star product $\star^{s . c}$ on $M$ (i.e. $\left.\int u \star^{s . c} v=\int u v\right)$.

Now intrinsically, the amplitude of the oscillating kernel defining the above strongly closed star product may be described geometrically as follows. Let

$$
\Phi: M \times M \times M \rightarrow M \times M \times M:(x, y, z) \mapsto(X, Y, Z)
$$

with

$$
s_{x} s_{y} s_{z}(X)=X, \quad Y=s_{z}(X), \quad Z=s_{y}(Y) .
$$

Now consider the Jacobian map of $\Phi$ :

$$
\operatorname{Jac}_{\Phi}(x, y, z):=\left|\frac{\partial(X, Y, Z)}{\partial(x, y, z)}\right| .
$$

Then, it turns out that in the above coordinate system the function $\mathrm{Jac}_{\Phi}$ depends only on the $a$ coordinates of the points and that its square root coincides with the above mentioned amplitude. More precisely, one has 
Theorem 8.10 [8] Let $\theta>0$. For $u$ and $v$ compactly supported functions on $M$, the formula

$$
u \star_{\theta}^{s . c} v:=\frac{1}{\theta^{2}} \int_{M \times M} \sqrt{\operatorname{Jac}_{\Phi}} e^{\frac{i}{\theta} S_{\text {can }}} u \otimes v
$$

extends to $L^{2}(M)$ as an associative product. The function algebra $\left(L^{2}(M), \star_{\theta}^{s . c}\right)$ is then a Hilbert algebra with respect to the natural Hilbert space structure on $L^{2}(M)$.

From this a continuous field of $C^{\star}$-algebras deforming $C_{0}(M)$ may be obtained via standard techniques (see e.g. [39] for the flat case and [5] for curved solvable symmetric spaces).

\section{References}

[1] R. Albuquerque, J. Rawnsley, Twistor Theory of Symplectic Manifolds J. Geometry and Physics 56 (2006) 214-246.

[2] P. Baguis, M. Cahen, A construction of symplectic connections through reduction, Lett. Math. Phys. 57 (2001), pp. 149-160.

[3] F. Bayen, M. Flato, C. Fronsdal, A. Lichnerowicz and D. Sternheimer, Quantum mechanics as a deformation of classical mechanics, Lett. Math. Phys. 1 (1977) 521-530 and Deformation theory and quantization, part I, Ann. of Phys. 111 (1978) 61-110.

[4] P. Bieliavsky, Espaces symétriques symplectiques, PhD thesis, ULB, Brussels, 1995.

[5] P. Bieliavsky, Strict quantization of solvable symmetric spaces, J. Symplectic Geom. 1 (2002), no. 2, 269-320.

[6] P. Bieliavsky, Ph. Bonneau, Y. Maeda, Universal Deformation Formulae, Symplectic Lie groups and Symmetric Spaces. math.QA/0308189

[7] P. Bieliavsky, M. Rooman, Ph. Spindel, Regular Poisson structures on massive non-rotating BTZ black holes. Nuclear Physics B 645, Issues 1-2, 25 (2002), 349-364. (hep-th/0206189).

[8] P. Bieliavsky, S. Detournay, Ph. Spindel, M. Rooman, Star products on extended massive nonrotating BTZ black holes. J. High Energy Phys. 2004, no. 6, 031

[9] P. Bieliavsky, Y. Maeda, Convergent star product algebras on "ax+b". Lett. Math. Phys. 62 (2002) 233-243. (math.QA/0209295).

[10] P. Bieliavsky, M. Massar, Oscillatory integral formulae for left-invariant star products on a class of Lie groups. Lett. Math. Phys. 58 (2001) 115-128.

[11] P. Bieliavsky, X. Tang, Y-J. Yao, Rankin-Cohen brackets and quantization of foliation, Part I: formal quantization, math.QA/0506506.

[12] S. Bochner, Curvature and Betti numbers, II, Ann. Math. 50 (1949) 77-93.

[13] F. Bourgeois, M. Cahen, A variational principle for symplectic connections, J. Geometry and Physics 30 (1999) 233-265. 
[14] R. Bryant, Two exotic holonomies in dimension four, path geometries, and twistor theory, Proc. Symp. in Pure Math. 53 (1991) 33-88.

[15] R. Bryant, Bochner-Kähler metrics, J. Amer. Math. Soc. 14 (2001) 623-715.

[16] C. Boubel, Symplectic connections with a parallel Ricci curvature, Proc. Edinburgh. Math. Soc. 46 (2003) $747-766$.

[17] M. Cahen, S. Gutt, Reduction, Induction and Ricci-flat symplectic connections, SG/050914.

[18] M. Cahen, S. Gutt and J. Rawnsley, Preferred invariant symplectic connections on compact coadjoint orbits, Lett. in Math. Phys. 48 (1999) 353-364.

[19] M. Cahen, S. Gutt and J. Rawnsley, Symplectic connections with parallel Ricci tensor, in Poisson Geometry, Banach Center Publications 51 (2000) 31-41.

[20] M. Cahen, S. Gutt, J. Horowitz and J. Rawnsley, Homogeneous symplectic manifolds with Riccitype curvature, J. Geom. Phys. 38 (2001) 140-151.

[21] M. Cahen, S. Gutt, L. Schwachhöfer, Construction of Ricci-type connections by reduction and induction, in The breadth of symplectic and Poisson Geometry, Marsden, J.E. and Ratiu, T.S. (eds), Progress in Math 232, Birkhauser, 2004. (math.DG/0310375)

[22] M. Cahen, L. Schwachhöfer, Special Symplectic Connections, math.DG/0402221.

[23] Q.-S. Chi, S.A. Merkulov, L. Schwachhöfer, On the Existence of Infinite Series of Exotic Holonomies, Inv. Math. 126, (1996) 391-411.

[24] A. Connes, M. Dubois-Violette, Noncommutative finite-dimensional manifolds. I. Spherical manifolds and related examples. Comm. Math. Phys. 230 (2002) 539-579.

[25] B.V. Fedosov, A simple geometrical construction of deformation quantization, J. Diff. Geom. 40 (1994) 213-238.

[26] M. Flato, A. Lichnerowicz and D. Sternheimer, Crochet de Moyal-Vey et quantification, C. R. Acad. Sci. Paris I Math. 283 (1976) 19-24.

[27] I. Gelfand, V. Retakh and M. Shubin, Fedosov Manifolds. dg-ga/9707024. Adv. Math. 136 (1998) $104-140$.

[28] S. Gutt, J. Rawnsley, Natural star products on symplectic manifolds and quantum moment maps, math.SG/0304498, Lett. Math. Phys. 66 (2003) 123-139.

[29] H. Hess, Connections on symplectic manifolds and geometric quantization. Springer Lecture Notes in Mathematics 836 (1980) 153-166.

[30] Y. Kamishima, Uniformization of Kähler manifolds with vanishing Bochner tensor, Acta Math. 172 (1994) 299-308.

[31] S. Kobayashi and K. Nomizu, Foundations of differential geometry. Vol II. John Wiley \& Sons, New York-London, 1963. 
[32] B. Kostant, Minimal coadjoint orbits and symplectic induction, in The breadth of symplectic and Poisson Geometry, Marsden, J.E. and Ratiu, T.S. (eds), Progress in Math 232, Birkhauser, 2004.

[33] A. Lichnerowicz, Déformations d'algèbres associées à une variété symplectique (les $*_{\nu}$-produits), Ann. Inst. Fourier, Grenoble 32 (1982) 157-209.

[34] D. McDuff, D. Salamon, Introduction to Symplectic Topology, Oxford Math. Monographs, Clarendon Press, Oxford 1998.

[35] S.A. Merkulov, L. Schwachhöfer, Classification of irreducible holonomies of torsion free affine connections, Ann. Math. 150 (1999) 77-149; Addendum: Classification of irreducible holonomies of torsion-free affine connections, Ann. Math. 150 (1999) 1177-1179.

[36] N.R. O'Brian and J.H. Rawnsley, Twistor Spaces, Ann. Global. Anal. Geom. 3 (1985) 29-58.

[37] R. Palais, The principle of symmetric criticality, Comm. Math. Phys. 69 (1979) 19-30.

[38] M. Panák, L. Schwachhöfer, Bochner-Kähler metrics and connections of Ricci type (preprint).

[39] M.A. Rieffel, Deformation quantization for actions of $R^{d}$. Mem. Amer. Math. Soc. 106 (1993), no. 506

[40] L. Schwachhöfer, On the classification of holonomy representations, Habilitations-schrift, Universität Leipzig (1998).

[41] L. Schwachhöfer, Homogeneous connections with special symplectic holonomy, Math. Zeit. 238 (2001) 655-688.

[42] L. Schwachhöfer, Connections with irreducible holonomy representations, Adv. Math. 160 (2001) $1-80$.

[43] M. Stiénon, À propos d'une structure complexe sur un espace de twisteurs pour certaines variétés symplectiques, Thèse (Bruxelles, 2004).

[44] P. Tondeur, Affine Zusammenhänge auf Mannigfaltigkeiten mit fast-symplektischer Struktur. Comment. Helv. Math. 36 (1961), 234-244.

[45] I. Vaisman, Symplectic Curvature Tensors. Monats. Math. 100 (1985) 299-327. See also: M. De Visher, Mémoire de licence, Bruxelles, 1999.

[46] I. Vaisman, Symplectic Twistor Spaces, J. Geometry and Physics 3 (1986) 507-524 and Variations on the theme of Twistor Spaces, Balkan J. Geom. Appl. 3 (1998) 135-156.

[47] A. Weinstein, Traces and triangles in symmetric symplectic spaces, Contemp. Math. 179 (1994) 261-270. 